\title{
A century of gray: A genomic locus found in 2 distinct Pseudomonas spp. is associated with historical and contemporary color defects in dairy products worldwide
}

\author{
Samuel J. Reichler, Nicole H. Martin, Rachel L. Evanowski, Jasna Kovac, ${ }^{*}$ Martin Wiedmann, \\ and Renato H. Orsi† \\ Milk Quality Improvement Program, Department of Food Science, Cornell University, Ithaca, NY 14853
}

\begin{abstract}
Some gram-negative bacteria, including Pseudomonas spp., can grow at refrigeration temperatures and cause flavor, odor, and texture defects in fluid milk. Historical and modern cases exist of gray and blue color defects in fluid milk due to Pseudomonas, and several recent reports have detailed fresh cheese spoilage associated with blue-pigment-forming Pseudomonas. Our goal was to investigate the genomes of pigmented Pseudomonas isolates responsible for historical and modern pigmented spoilage of dairy products in the United States to determine the genetic basis of pigment-forming phenotypes. We performed whole genome sequencing of 9 Pseudomonas isolates: 3 from recent incidents of graypigmented fluid milk (Pseudomonas fluorescens group), 1 from blue-pigmented cheese ( $P$. fluorescens group), 2 from a historical blue milk spoilage incident (Pseudomonas putida group), and 3 with no evidence for blue or gray pigment formation (2 from $P$. fluorescens group and 1 from Pseudomonas chlororaphis group). All 6 isolates collected from products with a gray or blue pigment defect were confirmed to produce pigment using potato dextrose agar or pasteurized milk. A subset of 2 isolates was selected for inoculation into milk and onto the surface of a model cheese for subsequent color measurement. These isolates produced different colors on potato dextrose agar, but produced nearly identical color defects in milk and on model cheese. For the same subset of 2 isolates, the gray color defect in milk was produced only in containers with ample headspace and not in full containers, suggesting that oxygen is vital for pigment formation. This work also demonstrated
\end{abstract}

Received December 20, 2018.

Accepted March 23, 2019.

*Current address: Department of Food Science, Pennsylvania State University, University Park 16802.

$\dagger$ Corresponding author: rho2@cornell.edu that a Pseudomonas isolate from cheese can produce a pigment defect in milk, and vice versa. Comparative genomics identified an accessory locus encoding tryptophan biosynthesis genes that was present in all isolates that produced gray or blue pigment under laboratory conditions and was only previously reported in $2 P$. fluorescens isolates responsible for blue mozzarella in Italy. Because this locus was found in genetically distant isolates belonging to different Pseudomonas species groups, it may have been acquired via horizontal gene transfer. These data suggest that several past and present gray- or blue-pigmented dairy spoilage events share a common genetic etiology that transcends species-level identification and merits further investigation to determine mechanistic details and modes of prevention.

Key words: Pseudomonas, gray, blue, milk, cheese

\section{INTRODUCTION}

Visual spoilage in dairy products has a high potential for economic harm to manufacturers. The relationship between food companies and their consumers has been revolutionized by social media, because consumers now have a platform on which to broadcast images and videos of spoiled or otherwise defective products. In several cases, attention was drawn to large-scale food spoilage incidents via social media (Karp, 2010; Stones, 2013), but this phenomenon is not isolated to large-scale events. The threat to a food manufacturer or brand from a single spectacularly spoiled unit of product cannot be overlooked or underestimated, particularly because a nontrivial proportion of consumers interpret food spoilage as a safety concern. In annual surveys performed by the Food Marketing Institute from 1993 to 1997, 41 to $69 \%$ of consumers volunteered spoilage-related answers when asked about the greatest threats to the safety of the food they ate (Food Marketing Institute, 1997). In similar surveys conducted in 1999 and 2000, 20 and 19\% of consumers volunteered spoiled or expired food as the single most important 
source of food poisoning (Food Marketing Institute, 2000). Although these surveys are somewhat old, they remain a reminder that significant dissonance may exist between manufacturer and consumer knowledge about the meaning of "spoilage," a topic beyond the scope of this publication. It suggests that consumers may misinterpret the severity of spoilage events or the consumption of spoiled food, particularly when recalls occur. A 2017 survey reported that $13 \%$ of consumers said they would never purchase a specific food product again once they were aware that it had been recalled, although the survey question did not specify a cause for the recall (Food Marketing Institute, 2017).

Pigmented bacteria have a long history of causing visual spoilage in dairy foods. References to blue milk in German and French agricultural and scientific literature date back over 200 yr (Borowski, 1788; Parmentier and Deyeux, 1799). In 1884, the first inoculations of blue-pigmented bacteria into sterilized milk were performed, and the bacteria produced a blue pigment in raw milk, a gray pigment in neutral $\mathrm{pH}$ sterilized milk, and a blue-gray pigment in sterilized acidified milk (Hueppe, 1884). Blue milk from the United States was first described by Duckwall (1905), who isolated the causative strain, Bacillus cyanogenus, from a milk sample in Pennsylvania. Upon inoculation into sterilized milk, this isolate produced no acid or coagulation, but colored the milk "slate-gray, which turns blue with acids." A 1914 report detailed an "outbreak" of blue milk in Iowa and included a detailed profile of the implicated organism, Bacillus cyanogenes (Hammer). Hammer described a gram-negative, motile, rod-shaped bacterium that produced a gray pigment in sterilized milk and a blue pigment in sterilized milk when cocultured with a lactic acid bacterium. Of note, although modern day Bacillus are exclusively gram-positive sporeformers, the generic name "Bacillus" was used more broadly in the past. These historical isolates no longer fall within the contemporary Bacillus genus.

Few reports exist of pigment defects in fluid milk in the last 100 years, which may be attributed to the widespread adoption of pasteurization for milk beginning in the early 20th century (Boor et al., 2017). A case of blue milk attributed to Pseudomonas fluorescens was reported in Germany in 2006 (Seiler, 2006), and a case of gray milk attributed to Pseudomonas azotoformans was reported by our group in 2017 (Evanowski et al., 2017). In both of these instances, pigment formation appeared to be dependent on the presence of oxygen.

There are also historical and contemporary reports of bacterial blue pigment in cheese. In the late 19th century, Bacillus cyaneo-fuscus contamination was blamed for blue Edam cheese in the Netherlands (Beyerinck, 1892), and blue cheese caused by Bacillus cyanogenus, the same organism that caused blue milk, was also reported (Conn, 1892). Pseudomonas fluorescens biovar IV caused blue-pigmented spoilage of queso fresco cheese from a processor in the United States in 2006 (Martin et al., 2011), and in 2010, 70,000 units of mozzarella cheese produced at a facility in Germany and sold in Italy turned blue and were confiscated by Italian officials (Greenhalgh, 2010). Again, P. fluorescens was responsible, and indigoidine was suggested as the pigment causing the defect (Cantoni and Bersani, 2010; Sechi et al., 2011; Cenci-Goga et al., 2014). Multilocus sequence typing identified a "blue branch" of strains in the P. fluorescens phylogeny (Andreani et al., 2015b; Chierici et al., 2016). Leucoindigoidine was found to be a chemical marker of blue spoilage potential (Caputo et al., 2015), and a high-molecular-weight indigo derivative, rather than indigoidine, was suggested as the identity of the pigment (Andreani et al., 2015a).

Some recent investigations of the Pseudomonas responsible for blue color defect formation have used next-generation sequencing [i.e., whole-genome sequencing (WGS) and RNA-seq] to provide hypotheses for the metabolic pathways leading to blue pigment formation in cheese and for the possible identity of the pigment. Comparative genomics performed on whole genome sequences of 4 Pseudomonas strains, 2 of which produced blue pigment in cheese, revealed that the pigmented strains possessed additional accessory copies of genes involved in the tryptophan biosynthesis pathway, whereas nonpigmented strains had only 1 copy of each gene (Andreani et al., 2015a). An RNA-seq analysis performed on the same set of isolates showed an upregulation of iron uptake (e.g., pyoverdine), oxidoreductase, and transcriptional regulation genes in the pigmented strains that was not present in the unpigmented strains. Transposon-induced pigment-deficient mutants of Ps_77, a blue-pigment-producing strain of P. fluorescens isolated from beef, confirmed the involvement of the accessory copy of the tryptophan biosynthesis gene $\operatorname{trp} B$ in production of the blue phenotype (Andreani, 2016). Transposon-induced mutations in genes putatively involved in oxidative stress response, biofilm formation, and cell signaling were also able to eliminate the production of the blue pigment.

Our goal in this study was to investigate the gray- and blue-pigment-producing strains of Pseudomonas from our own culture collection, as well as publicly available blue-pigmented strains, to aid in the global effort to understand the mechanisms of gray and blue pigment production and ultimately work to prevent their formation in dairy products. To this end, we performed WGS and phenotypic evaluations on 3 recently obtained isolates of Pseudomonas from pasteurized milk with a gray defect, 1 recently obtained isolate from blue-pigmented 
fresh cheese, 3 recently obtained isolates unassociated with these defects, and 2 isolates responsible for the historic production of blue and gray pigments in milk.

\section{MATERIALS AND METHODS}

\section{Isolate Selection and Growth Conditions}

We used Pseudomonas strains with and without pigmented phenotypes for comparative genomics. We selected 7 representative Pseudomonas strains that had been identified previously by partial sequencing of their 16S rDNA from the Milk Quality Improvement Program isolate collection (Table 1). These included 4 strains that had previously shown evidence of causing blue or gray color defects in dairy products and 3 that did not. The 3 isolates with no prior evidence for blue or gray pigment formation originated from dairy products or dairy processing environments: R5-0199 was selected specifically for its close partial $16 \mathrm{~S}$ rDNA sequence match with the 4 recently obtained $P$. fluorescens group isolates associated with color defects. Additional details on the history and characterization of these isolates can be accessed via Food Microbe Tracker (http://www .foodmicrobetracker.com; Vangay et al., 2013).

Additionally, 2 Pseudomonas isolates were obtained from the USDA Agricultural Research Service Northern Regional Research Laboratory Culture Collection (https://nrrl.ncaur.usda.gov/). These 2 isolates, B-251 and B-252, were given laboratory IDs of FSL R103386 and FSL R10-3389, respectively (Table 1). Both isolates are reported to originate from unpasteurized blue milk obtained in Iowa in October 1913 (Hammer, 1914; Haynes, 1961). The lyophilized cultures were rehydrated in $2 \mathrm{~mL}$ of brain heart infusion (BHI) broth (BD Diagnostics, Sparks, MD), which was immediately inoculated onto BHI agar plates (BD Diagnostics). The plates were incubated at $28^{\circ} \mathrm{C}$ for $24 \mathrm{~h}$. A single colony from each of these plates was inoculated onto a new BHI agar plate, which was incubated at $28^{\circ} \mathrm{C}$ for $24 \mathrm{~h}$. An isolated colony from each of these plates was inoculated into $5 \mathrm{~mL}$ of BHI broth, and the broth was then incubated at $32^{\circ} \mathrm{C}$ for $18 \mathrm{~h}$ before freezing at $-80^{\circ} \mathrm{C}$ in BHI broth supplemented with $15 \%$ glycerol for long-term storage.

For the milk and cheese inoculation experiments and for the WGS experiments described in the subsections that follow, the isolates were recovered from stocks of BHI broth with $15 \%$ glycerol stored at $-80^{\circ} \mathrm{C}$. The stocks were removed from storage, and a loopful of each was streaked onto BHI agar plates before the plates were incubated at $32^{\circ} \mathrm{C}$ for $48 \mathrm{~h}$. A colony of each isolate was selected and inoculated into $5 \mathrm{~mL}$ of BHI broth, and the broth was then incubated without

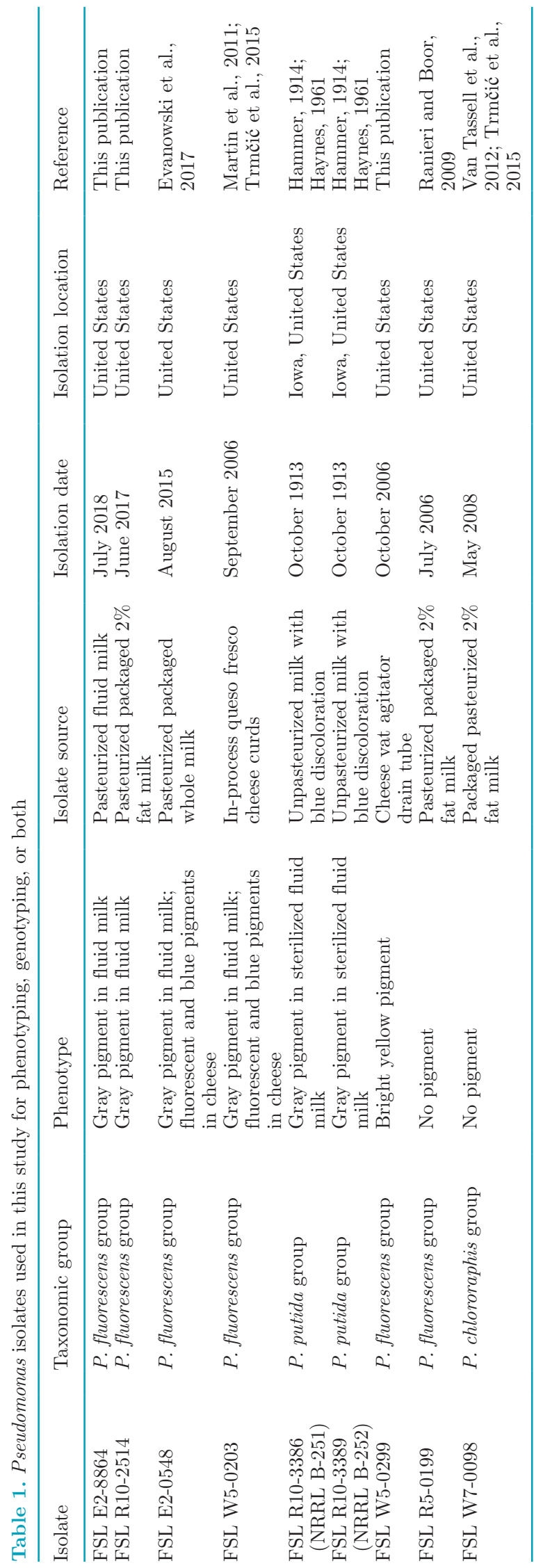

Journal of Dairy Science Vol. 102 No. 7, 2019 
shaking at $32^{\circ} \mathrm{C}$ for 18 to $24 \mathrm{~h}$ to produce a bacterial suspension of approximately $8 \mathrm{log} \mathrm{cfu} / \mathrm{mL}$.

\section{Initial Color Defect Screening}

To initially characterize pigment production for the 6 isolates that had previously been reported to produce blue or gray pigmentation, of our 9 total Pseudomonas isolates, we recovered isolates FSL E2-0548, FSL E28864, FSL R10-2514, FSL R10-3386, FSL R10-3389, and FSL W5-0203 from frozen glycerol stocks as described above, and a single colony from the BHI plate was streaked onto 2 sets of potato dextrose agar (PDA; BD Diagnostics) plates. The first set of plates was incubated at $6^{\circ} \mathrm{C}$ for $12 \mathrm{~d}$, and the second set was incubated at $21^{\circ} \mathrm{C}$ for $5 \mathrm{~d}$. Every $24 \mathrm{~h}$ during incubation, the plates were removed from their respective incubators and photographed using an EOS Rebel T3 DSLR camera (Canon, Tokyo, Japan) with the following settings: f/5.6, 1/60 s exposure, ISO-800, $55 \mathrm{~mm}$ focal length, compulsory flash, and fluorescent white balance.

Based on the results of the screening on PDA, we performed further screening of the 6 isolates selected above, along with FSL R5-0199, an unpigmented strain, in pasteurized milk. Pasteurized, homogenized $2 \%$ fat milk in $473-\mathrm{mL}$ (16 US fluid ounce) containers was purchased from a local supermarket, and half of the milk (approximately $230 \mathrm{~mL}$ ) was aseptically removed from each container. We prepared 18-h bacterial suspensions of the selected isolates as described previously, and a 1 -mL aliquot of each was centrifuged at $18,000 \times g$ for $1 \mathrm{~min}$. The supernatants were removed, and the pellets were resuspended in $1 \mathrm{~mL}$ of pasteurized milk. Each 1-mL bacterial suspension was then added to a prepared half-full container of milk. The containers were sealed, gently inverted to mix their contents, and incubated at $21^{\circ} \mathrm{C}$ for $96 \mathrm{~h}$. After 48,72 , and $96 \mathrm{~h}$, photographs were taken using the same camera settings as described above for the PDA photographs, but without the camera flash.

\section{Milk and Cheese Color Defect Assessment}

Based on the results of the preliminary screenings performed on PDA and in milk, we selected a subset of 2 pigment-producing isolates (FSL E2-0548 and FSL W5-0203) and 1 negative control isolate (FSL W50299) for further analysis of pigment formation in milk and on cheese. We specifically selected FSL E2-0548 and FSL W5-0203 because they originated from different matrices (fluid milk and cheese), and because they exhibited different color phenotypes on PDA at $6^{\circ} \mathrm{C}$. Selection of these 2 isolates also allowed us to test the hypothesis that the same isolates can cause color defects in both cheese and fluid milk. We selected FSL W5-0299 as the negative control because it did not exhibit blue or gray pigmentation on cheese, but yellow (N. H. Martin, unpublished data). Milk samples for color defect assessment were produced as described by Evanowski et al. (2017). Briefly, a 1-mL aliquot of an overnight bacterial suspension of each isolate was pelleted by centrifugation and resuspended and serially diluted in PBS (Weber Scientific, Hamilton, NJ). To inoculate, $500 \mu \mathrm{L}$ of each appropriate dilution was added to 2 half-filled $946-\mathrm{mL}$ (1 US quart) high-density polyethylene containers of pasteurized, homogenized $2 \%$ fat milk (Cornell University Dairy, Ithaca, NY) and $1 \mathrm{~mL}$ of each appropriate dilution was added to 2 completely filled 946-mL high-density polyethylene containers of pasteurized, homogenized $2 \%$ fat milk. This achieved an initial inoculum concentration of approximately 3.75 $\log \mathrm{cfu} / \mathrm{mL}$ in both the filled and half-filled containers. We determined SPC by spiral plating (Autoplate 5000; Advanced Instruments, Norwood, MA) immediately after inoculation and after $14 \mathrm{~d}$ of storage at $6^{\circ} \mathrm{C}$ to verify the growth of the inoculum. We selected an incubation temperature of $6^{\circ} \mathrm{C}$ for both the milk and the cheese, because this represented the semi-abusive conditions that dairy products may realistically encounter in the homes of consumers (Kosa et al., 2007). To determine color change over time, we applied the approach used by Evanowski et al. (2017). Briefly, sample L*a*b* coordinates were measured immediately after inoculation and after $14 \mathrm{~d}$ of storage using a HunterLab ColorQuest XE colorimeter (Hunter Associates Laboratory, Reston, VA). In the $\mathrm{L}^{*} \mathrm{a}^{*} \mathrm{~b}^{*}$ color space, $\mathrm{L}^{*}$ represents lightness $\left(L^{*}=0\right.$ is black; $L^{*}=100$ is a diffuse white), a* represents the position between green and red (negative values are green; positive values are red), and $b^{*}$ represents the position between blue and yellow (negative values are blue; positive values are yellow).

We used laboratory-scale mozzarella-style cheese made from $1.9 \mathrm{~L}$ (0.5 US gallon) of locally obtained pasteurized, homogenized whole milk for color defect assessment. The cheese was prepared as described previously by Carroll (2002). The milk was heated to $13^{\circ} \mathrm{C}$, and $60 \mathrm{~mL}$ of $360 \mathrm{mM}$ citric acid solution (Fisher Chemical, Fair Lawn, NJ) was stirred in. The milk was further heated to $32^{\circ} \mathrm{C}$, and a solution containing 616 $\mu \mathrm{L}$ of prepared vegetable rennet (mucorpepsin; New England Cheesemaking Supply Company, South Deerfield, MA) in $30 \mathrm{~mL}$ of deionized water was added with gentle stirring. The milk was allowed to coagulate without stirring for $5 \mathrm{~min}$ while heating to $40^{\circ} \mathrm{C}$ before the curd was drained. The curd was then heated in a 1,100 $\mathrm{W}$ microwave oven set at full power for $1 \mathrm{~min}$, followed by kneading with gloved hands. Next, the curd was heated for $30 \mathrm{~s}$ and kneaded for a second time. Finally, 
the curd was heated for $30 \mathrm{~s}$, and $1.18 \mathrm{~g}$ of granulated sodium chloride (Fisher Chemical) was added before kneading again. The resulting molten cheese was flattened into a disk approximately $1 \mathrm{~cm}$ in height before it was chilled in an ice-water bath and stored at $4^{\circ} \mathrm{C}$ in a sealed plastic bag to await inoculation. To inoculate, 1-mL bacterial suspensions prepared from overnight cultures of isolates FSL E2-0548, FSL W5-0203, and FSL W5-0299, were pelleted in a microcentrifuge at $18,000 \times g$ for $1 \mathrm{~min}$ and resuspended in $1 \mathrm{~mL}$ of PBS. Pieces of the prepared cheese approximately $4.5 \mathrm{~cm}$ in diameter and $1 \mathrm{~cm}$ in height were placed into sterile 90-mm-diameter polystyrene Petri dishes and spotinoculated with $50 \mu \mathrm{L}$ of the bacterial suspensions. The dishes were covered and stored at $6^{\circ} \mathrm{C}$ for $7 \mathrm{~d}$. At $2 \mathrm{~d}$ and $7 \mathrm{~d}$ after inoculation, photographs of the cheese samples were taken using an EOS Rebel T3 DSLR camera (Canon) in a darkened room and illuminated by (1) the camera flash (f/5.6, 1/60 s exposure, ISO-800, 55 $\mathrm{mm}$ focal length) and (2) a $6 \mathrm{~W}, 365$-nm wavelength UV-A lamp (VWR, Radnor, PA) with full automatic camera settings. We performed color analysis of the photographed cheese samples essentially as described by Yam and Papadakis (2004). Briefly, the pigmented area of the cheese (or the entire surface of the negative control cheese) was selected in Photoshop (CC 2018; Adobe Systems, San Jose, CA), and the histogram window was used to obtain the mean, standard deviation, and median L, a, and b values for the selected area, which were then converted to standard $\mathrm{L}^{*}, \mathrm{a}^{*}$, and $\mathrm{b}^{*}$ coordinates. For both the inoculated milk and cheese, $\mathrm{L} * \mathrm{a} * \mathrm{~b} *$ coordinates across strains and treatments were visualized using ggplot2 (Wickham, 2016).

\section{DNA Extraction and Whole-Genome Sequencing}

Extraction of genomic DNA and WGS was performed using select modifications of the procedure described by
Kovac et al. (2016) for all 9 Pseudomonas isolates listed in Table 1. Briefly, genomic DNA was extracted and purified from the prepared bacterial suspensions using a QIAamp DNA MiniKit (Qiagen, Valencia, CA) and checked for concentration and purity using a Nanodrop spectrophotometer and a Qubit dsDNA high sensitivity kit (both ThermoFisher Scientific, Waltham, MA). We submitted DNA samples to the Cornell University Institute of Biotechnology Genomics facility, where libraries were prepared using the Nextera XT DNA Library Preparation Kit (Illumina, San Diego, CA). Samples were pooled and sequenced in 2 Illumina HiSeq 2500 rapid runs with $2 \times 100$-bp paired-end reads or in an Illumina MiSeq with $2 \times 250$-bp paired-end reads. The detailed protocol for genomic DNA extraction and quality analysis for WGS can be accessed at https:// cornell.box.com/v/Reichler2018b.

\section{Quality Checks, Genome Assembly, Genome Annotation, and Assessment of Orthology}

Raw sequence reads were checked for quality using FASTQC v 0.11 .5 (https://www.bioinformatics .babraham.ac.uk/projects/fastqc/) and trimmed using Trimmomatic version 0.36 (Bolger et al., 2014). After trimming, sequence reads were rechecked for quality and then used for de novo genome assembly. Genomes were assembled using SPAdes version 3.6.2 or 3.10.1 (Bankevich et al., 2012) using the "-careful" parameter. Final draft assemblies were annotated using Prokka v 1.12 (Seemann, 2014). Orthologs were identified using the program Roary (Page et al., 2015). An identity threshold of $50 \%$ was used to account for intrinsic diversity in the isolate set. Whole genome assemblies were deposited in GenBank (https://www.ncbi.nlm .nih.gov/genbank/) under BioProject PRJNA341280. Accession IDs for individual isolates can be found in Table 2.

Table 2. Summary of Pseudomonas whole-genome metrics

\begin{tabular}{|c|c|c|c|c|c|c|}
\hline Strain & $\begin{array}{l}\text { Genome size }{ }^{1} \\
\text { (bp) }\end{array}$ & $\% \mathrm{GC}$ & $\begin{array}{l}\text { No. of } \\
\text { contigs }\end{array}$ & $\mathrm{N} 50^{2}$ & $\begin{array}{l}\text { Average } \\
\text { coverage }\end{array}$ & GenBank accession ID \\
\hline FSL E2-8864 & $6,755,291$ & 59.8 & 188 & 95,881 & 23 & RWKC00000000 \\
\hline FSL R10-2514 & $6,397,553$ & 59.9 & 57 & 260,661 & 108 & RWKD00000000 \\
\hline FSL W5-0203 & $6,284,734$ & 59.7 & 114 & 136,285 & 177 & RXHY00000000 \\
\hline FSL R10-3386 & $6,068,646$ & 62.6 & 91 & 175,121 & 46 & RWKE00000000 \\
\hline FSL R10-3389 & $6,087,214$ & 62.5 & 87 & 175,140 & 43 & RWKF00000000 \\
\hline FSL W7-0098 & $5,026,399$ & 58.3 & 35 & 449,785 & 145 & RXHW00000000 \\
\hline
\end{tabular}

${ }^{1}$ This represents the combined size of all contigs.

${ }^{2} \mathrm{~N} 50=$ weighted median statistic such that $50 \%$ of the entire assembly is contained in contigs or scaffolds of a length equal to or larger than this value. 


\section{Phylogenetic Analysis}

We obtained the 16S rDNA sequences of 164 Pseudomonas type strains from the Ribosomal Database Project (Cole et al., 2009). The 16S rDNA sequences from the 9 newly sequenced Pseudomonas isolates in this study were extracted from their whole genome sequences using RNAmmer (Lagesen et al., 2007). These sequences were aligned using MUSCLE (Edgar, 2004) and used to generate a maximum-likelihood tree in RAxML (Stamatakis, 2014) with the General Time Reversible nucleotide substitution model, an optimization of substitution rates (gamma model of rate heterogeneity), and estimation of proportions of invariable sites. To measure node confidence, 1,000 bootstraps were generated.

Whole genome sequence assemblies of 52 Pseudomonas type strains (i.e., denoted as type strains by the American Type Culture Collection, Deutsche Sammlung von Mikroorganismen und Zellkulturen, or other collections) were downloaded from http://www .pseudomonas.com. We also obtained 4 whole genome sequences of pigmented and unpigmented Pseudomonas strains (Ps_20, Ps_22, Ps_40, and Ps_77; generated by Andreani et al. (2015a) from GenBank. The Ps_22 and Ps_77 strains were isolated in Italy and the United Kingdom, respectively, and are associated with blue color defects similar to those observed for FSL W5-0203. The Ps_20 and Ps_40 strains were 2 isolates that did not produce the color defect and were used for comparison with Ps_22 and Ps_77. We used these sequences along with the whole genome sequences generated in this study to construct an Average Nucleotide Identity by Basic Local Alignment Search Tool (ANIb) distance matrix using pyani (https://github .com/widdowquinn/pyani). This distance matrix was visualized as a dendrogram using the ggdendro package (de Vries and Ripley, 2016) in R (R Core Team, 2018). To confirm the phylogeny of a subset of closely related isolates (5 newly sequenced isolates, 4 previously sequenced isolates, type strain Pseudomonas lactis DSM 29167, and type strain Pseudomonas paralactis DSM 29164) with higher resolution than the ANIb dendrogram, we constructed a maximum parsimony tree from their pan-genome SNPs using kSNP3 (Gardner et al., 2015) with a k-mer size of 19.

Predicted amino acid sequences annotated as the $\operatorname{trp} A, \operatorname{trp} B, \operatorname{trp} C, \operatorname{trp} D$, and $\operatorname{trp} F$ genes were extracted from the 9 newly sequenced genomes and the 4 previously sequenced genomes. These sequences were used to construct maximum-likelihood evolutionary trees for each gene based on the Jones-Taylor-Thornton matrixbased model (Jones et al., 1992) implemented in MEGA
X (Kumar et al., 2018). We used a discrete Gamma distribution with 5 categories to model evolutionary rate differences among sites; all positions containing gaps and missing data were eliminated, and 1,000 bootstrap replicates were performed.

\section{Hidden Markov Models}

We downloaded hidden Markov models for proteins hypothesized to be involved in the biosynthesis of pyoverdine, pyocyanin, tryptophan, indigoidine, violacein, and blue copper-binding proteins (see Supplemental Table S1 for models included; https://doi.org/10.3168/ jds.2018-16192) from the Pfam database (Finn et al., 2016). We used these models to search for their targeted protein families or domains in the annotated predicted protein sequences of the 9 newly sequenced Pseudomonas isolates using HMMER v. 3.1b2 (http:// hmmer.org) in maximum sensitivity mode. An E-value of $<1 \times 10^{-3}$ for one or more predicted proteins from an isolate was considered significant evidence of the presence of a given protein family or domain for that isolate.

\section{RESULTS}

\section{Color Defect Screening on PDA and in Milk}

We initially screened the 6 isolates associated with either a gray color defect in milk or a blue color defect in cheese for color formation on PDA (incubated at 6 or $21^{\circ} \mathrm{C}$ ) and in pasteurized milk. On PDA incubated at $21^{\circ} \mathrm{C}$, 4 isolates (FSL E2-8864, FSL E2-0548, FSL W5-0203, and FSL R10-2514) showed clearly visible color development after $3 \mathrm{~d}$ of incubation, whereas the other 2 isolates showed either no color development (FSL R10-3389) or very weak orange coloration (FSL R10-3386) after 3 and $5 \mathrm{~d}$ (Figure 1). The 4 isolates with clear color development showed varied intensities of gray or gray-orange to gray-blue color; isolate FSL E2-8864 showed the weakest color (defined by the http: //html-color-codes.info web tool as gray-orange after both $3 \mathrm{~d}$ and $5 \mathrm{~d}$ of incubation) and FSL W5-0203 showed the darkest color (defined as dark gray-blue after both $3 \mathrm{~d}$ and $5 \mathrm{~d}$ of incubation). Consistent with the data obtained for the same group of isolates at $21^{\circ} \mathrm{C}$, incubation of isolates on PDA at $6^{\circ} \mathrm{C}$ also identified a group of 4 isolates (FSL E2-8864, FSL E2-0548, FSL W5-0203, and FSL R10-2514) with clear color development after $12 \mathrm{~d}$ of incubation and a group of 2 isolates (FSL R10-3386 and FSL R10-3389) with very weak growth after $12 \mathrm{~d}$ at $6^{\circ} \mathrm{C}$ and no evidence of color formation (Figure 2). Interestingly, the 4 isolates with 


Isolate $1 \mathrm{~d}$.
FSL E2-8864
FSL R10-2514
FSL E2-0548
FSL W5-0203
FSL R10-3386 R10-3389
(NRRL B-251)

Figure 1. Growth and color phenotype of pigment-associated isolates of Pseudomonas on potato dextrose agar after incubation at $21^{\circ} \mathrm{C}$ for 1,3 , and $5 \mathrm{~d}$.

clear color development showed a wider range of color when incubated at $6^{\circ} \mathrm{C}$ than when incubated at $21^{\circ} \mathrm{C}$ : colors displayed at $6^{\circ} \mathrm{C}$ ranged from light gray-violet (FSL E2-8864) and dark gray-blue (FSL R10-2514) to very dark reddish-brown (FSL E2-0548) and dark blue (FSL W5-0203). Color formation was evident for FSL R10-2514 after 3 d incubation and for FSL E2-8864, FSL E2-0548, and FSL W5-0203 by $6 \mathrm{~d}$ incubation. Over time, the color on the plates intensified but did not appear to change from one color to another (e.g., from blue to brown).
All 6 isolates additionally showed evidence of gray pigment formation in pasteurized milk after $3 \mathrm{~d}$ at $21^{\circ} \mathrm{C}$ (Figure 3). As well, FSL E2-8864, FSL R10-2514, FSL E2-0548, and FSL W5-0203 exhibited fluorescence under UV light (Supplemental Figure S1; https://doi .org/10.3168/jds.2018-16192). Extensive proteolysis, evidenced by coagulation and separation of the milk samples, appeared after $3 \mathrm{~d}$ of incubation and became more pronounced after $4 \mathrm{~d}$ of incubation. An uninoculated milk sample showed neither color change nor coagulation after $4 \mathrm{~d}$ incubation. 


Isolate
FSL E2-8864

Figure 2. Growth and color phenotype of pigment-associated isolates of Pseudomonas on potato dextrose agar after incubation at $6^{\circ} \mathrm{C}$ for 3,6 , and $12 \mathrm{~d}$.

\section{Color Defect Demonstration and Measurement in Milk and Cheese}

We selected 2 blue- or gray-pigmented isolates and 1 yellow-pigmented isolate for more detailed analysis of color defects produced in milk and on cheese. All 3 isolates were inoculated into filled and half-filled containers of pasteurized, homogenized, $2 \%$ milk followed by incubation at $6^{\circ} \mathrm{C}$ for $14 \mathrm{~d}$. Milk from full containers that were inoculated with FSL E2-0548 or FSL W5-0203 were not substantially different in $\mathrm{L}^{*} \mathrm{a} \mathrm{b}^{*}$ coordinates from uninoculated milk in half-full or full containers, or from milk inoculated with the control strain FSL W5-0299 in half-full or full containers. This indicated no detectable color change (Figure 4a). However, halffull containers inoculated with FSL E2-0548 and FSL W5-0203 exhibited decreased values in $\mathrm{L}^{*} \mathrm{a} \mathrm{b}^{*}$ relative to the uninoculated half-full container control. These decreased values indicated that these samples were darker, greener, and less yellow than the uninoculated negative control sample. Only small differences existed between the $\mathrm{L}^{*} \mathrm{a}^{*} \mathrm{~b}^{*}$ coordinates of FSL E2-0548 and FSL W5-0203, but all coordinate values were numerically lower for FSL E2-0548 than they were for FSL 
W5-0203. Half-full and full containers of milk inoculated with these 3 Pseudomonas isolates showed SPC between 7.3 and $8.5 \log \mathrm{cfu} / \mathrm{mL}$ after $14 \mathrm{~d}$ of incubation (data not shown). All isolates reached a higher plate count in half-filled containers than they did in full containers, and the population difference between full and half-full containers ranged from 0.3 to $1.1 \log \mathrm{cfu} / \mathrm{mL}$. The SPC of the full and half-full uninoculated control containers did not increase over the $14 \mathrm{~d}$ of incubation.

When the same 3 isolates were inoculated onto the surface of mozzarella-style cheese followed by incubation at $6^{\circ} \mathrm{C}$ for $2 \mathrm{~d}$, blue pigment was present on the cheeses inoculated with FSL E2-0548 and FSL W5-0203 when viewed under white light, and the pigmented areas fluoresced under UV light (Figure 5). The cheese inoculated with the control strain FSL W5-0299 and the uninoculated (negative control) cheese showed no visible pigmentation under white light and did not fluoresce under UV light after $2 \mathrm{~d}$. After $7 \mathrm{~d}$ of incubation, dark gray-blue pigmentation was visible on the cheeses inoculated with FSL E2-0548 and FSL W5-0203 under white light, but only the pigmented areas on the cheese inoculated with FSL W5-0203 fluoresced under UV light. The cheese inoculated with FSL W5-0299 had a yellow pigment after $7 \mathrm{~d}$ of incubation, and the pigmented areas fluoresced under UV light.
The pigmented regions of photographs of the cheese inoculated with FSL E2-0548 and FSL W5-0203 both exhibited decreased values of $\mathrm{L}^{*} \mathrm{a}^{*} \mathrm{~b}^{*}$ when compared with the negative control, indicating that they were darker, greener, and less yellow than the control cheese (Figure 4b). Cheese inoculated with FSL E2-0548 was lower in $\mathrm{L}^{*}$ and $\mathrm{b}^{*}$ and higher in $\mathrm{a}^{*}$ than cheese inoculated with FSL W5-0203, indicating that FSL E2-0548 produced a pigment that was darker, less green, and bluer than that produced by FSL W5-0203. The pigmented regions of cheese inoculated with FSL W5-0299 exhibited a decreased value of $\mathrm{L}^{*}$ and increased values of $a^{*}$ and $b^{*}$ compared with the negative control, indicating that they were darker, redder, and yellower than the control cheese.

\section{WGS Metrics}

The WGS of the 9 isolates yielded genomes that ranged from 5,026,339 bp (FSL W7-0098) to 7,139,722 bp (FSL W5-0299; Table 2). The GC content ranged from $58.3 \%$ to $62.6 \%$. The average coverage achieved was lowest for FSL E2-8864 with $23 \times$ and highest for FSL W5-0203 with $177 \times$. The number of contigs in the final high-quality draft genomes ranged from 35 (FSL W7-0098) to 260 (FSL E2-0548), and the N50 (defined

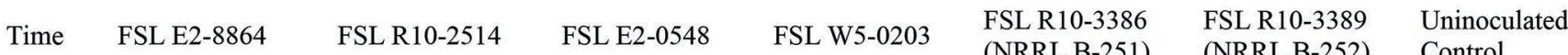

$\begin{array}{llll}\text { (NRRL B-251) } & \text { (NRRL B-252) Control }\end{array}$

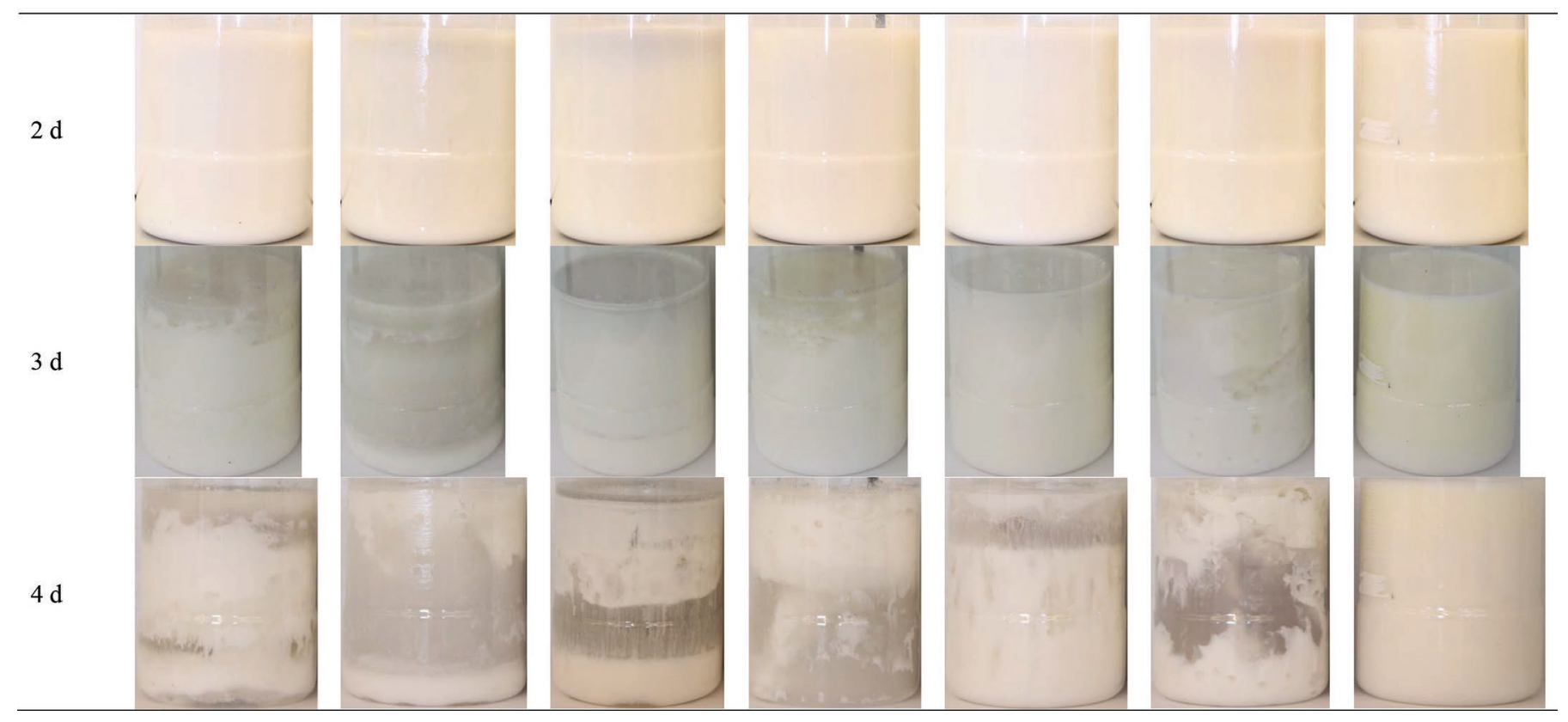

Figure 3. Growth of pigment-associated isolates of Pseudomonas in pasteurized, homogenized $2 \%$ fat milk after incubation in half-filled containers at $21^{\circ} \mathrm{C}$ for 2,3 , and $4 \mathrm{~d}$. 
as a weighted median statistic such that $50 \%$ of the entire assembly is contained in contigs or scaffolds of a length equal to or larger than this value) ranged from 74,043 bp (FSL E2-0548) to 194,733 bp (FSL R5-0199).

\section{Phylogenetic Analysis of 16S rDNA Sequences}

Initial phylogenetic characterization of the 6 Pseudomonas isolates previously linked to blue or gray color defects in dairy products and that were characterized by the initial tests on PDA and in fluid milk showed that these isolates represented 2 distinct clusters in a $16 \mathrm{~S}$ rDNA phylogenetic tree (Supplemental Figure S2; https://doi.org/10.3168/jds.2018-16192). Of these isolates, 4 (FSL W5-0203, FSL E2-8864, FSL R10-2514, and FSL E2-0548) represented a monophyletic clade that clustered with the $16 \mathrm{~S}$ rDNA sequence for the $P$. azotoformans type strain. The other 2 isolates previ-
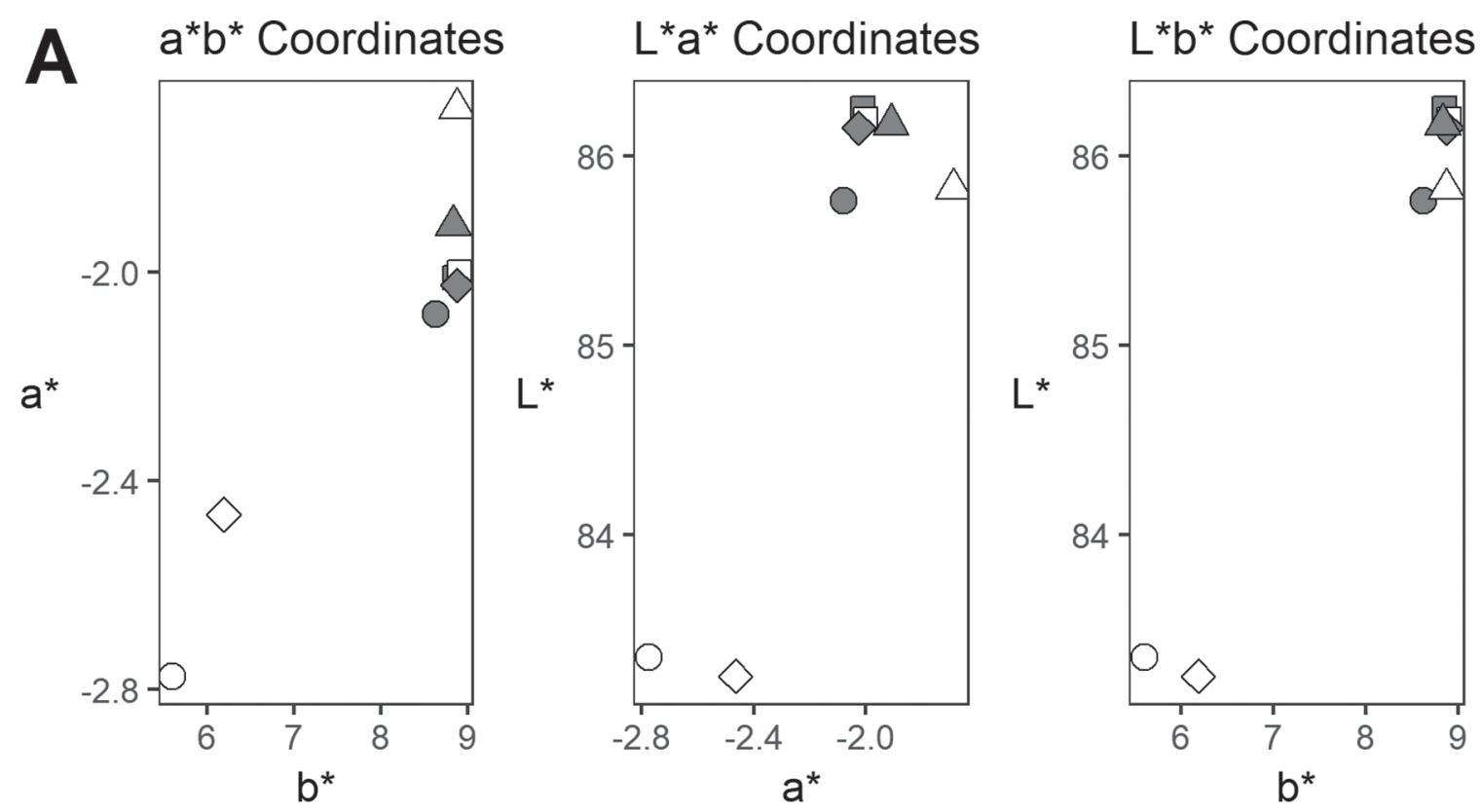

Isolate

Uninoculated

E2-0548

$\diamond W 5-0203$

W5-0299
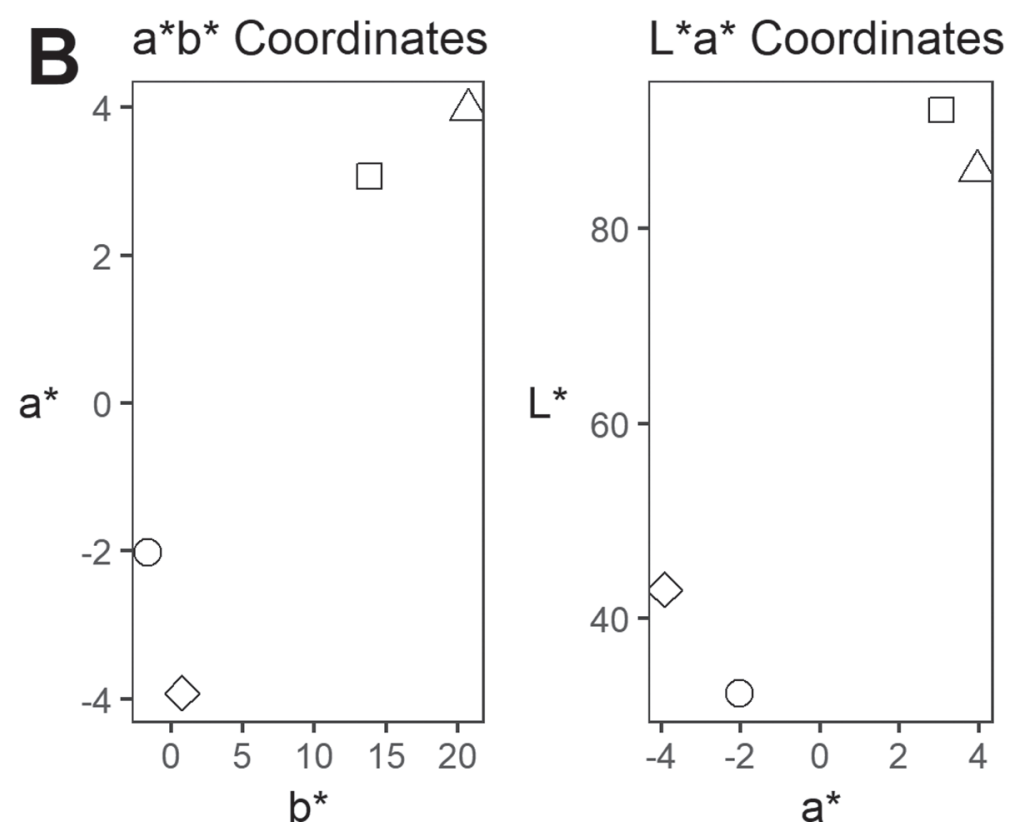

\section{$L^{*} b^{*}$ Coordinates}
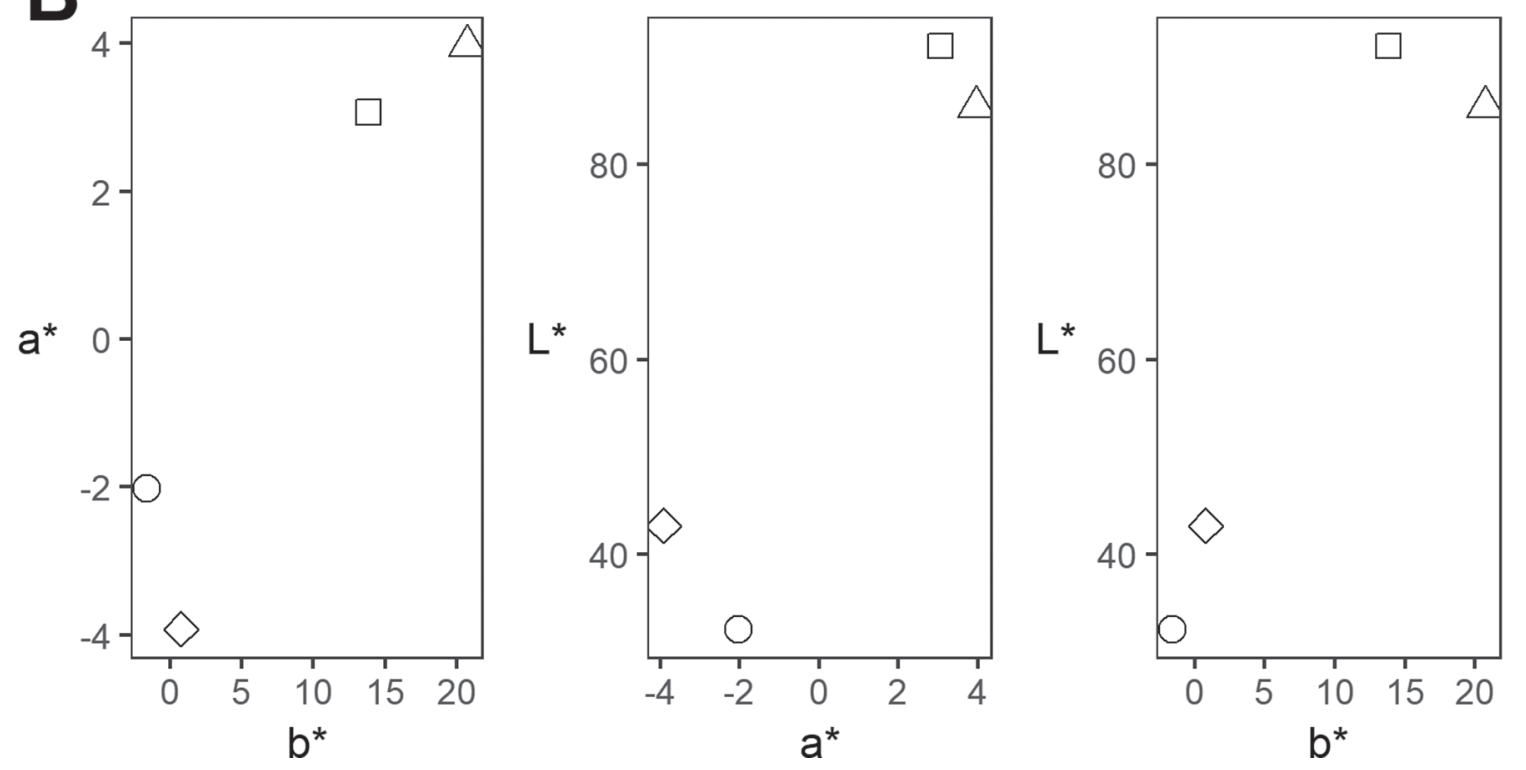

Container

Full

Half-full

Figure 4. Color (expressed as L*a*b* coordinates; $\mathrm{L}^{*}=$ lightness, $\mathrm{a}^{*}=$ red-green color, $\mathrm{b}^{*}=$ yellow-blue color) of $(\mathrm{A})$ pasteurized fluid milk after inoculation with pigment-associated strains of Pseudomonas and incubation at $6^{\circ} \mathrm{C}$ for $14 \mathrm{~d}$, and (B) mozzarella-style cheese after inoculation with pigment-associated strains of Pseudomonas and incubation at $6^{\circ} \mathrm{C}$ for $7 \mathrm{~d}$. 
ously linked to blue or gray color defects in dairy products (FSL R10-3386 and FSL R10-3389) also clustered together but were very distinct from the clade described above and formed a monophyletic clade with the $P$. putida type strain. Low bootstrap support $(<15 \%)$ was generally achieved throughout the tree, which suggests that the $16 \mathrm{~S}$ sequences may not provide enough resolution to clearly delineate Pseudomonas species, including among the isolates characterized here.
The 3 control isolates, which had not been linked to blue or gray color defects and were included in our WGS to allow for comparative genomics approaches, were also included in the $16 \mathrm{~S}$ rDNA phylogeny. Among these 3 isolates, FSL R5-0199 grouped into the same monophyletic clade that included the $P$. azotoformans type strain and the 4 Pseudomonas isolates previously linked to blue or gray color defects in dairy products. The other 2 control isolates clustered separately from

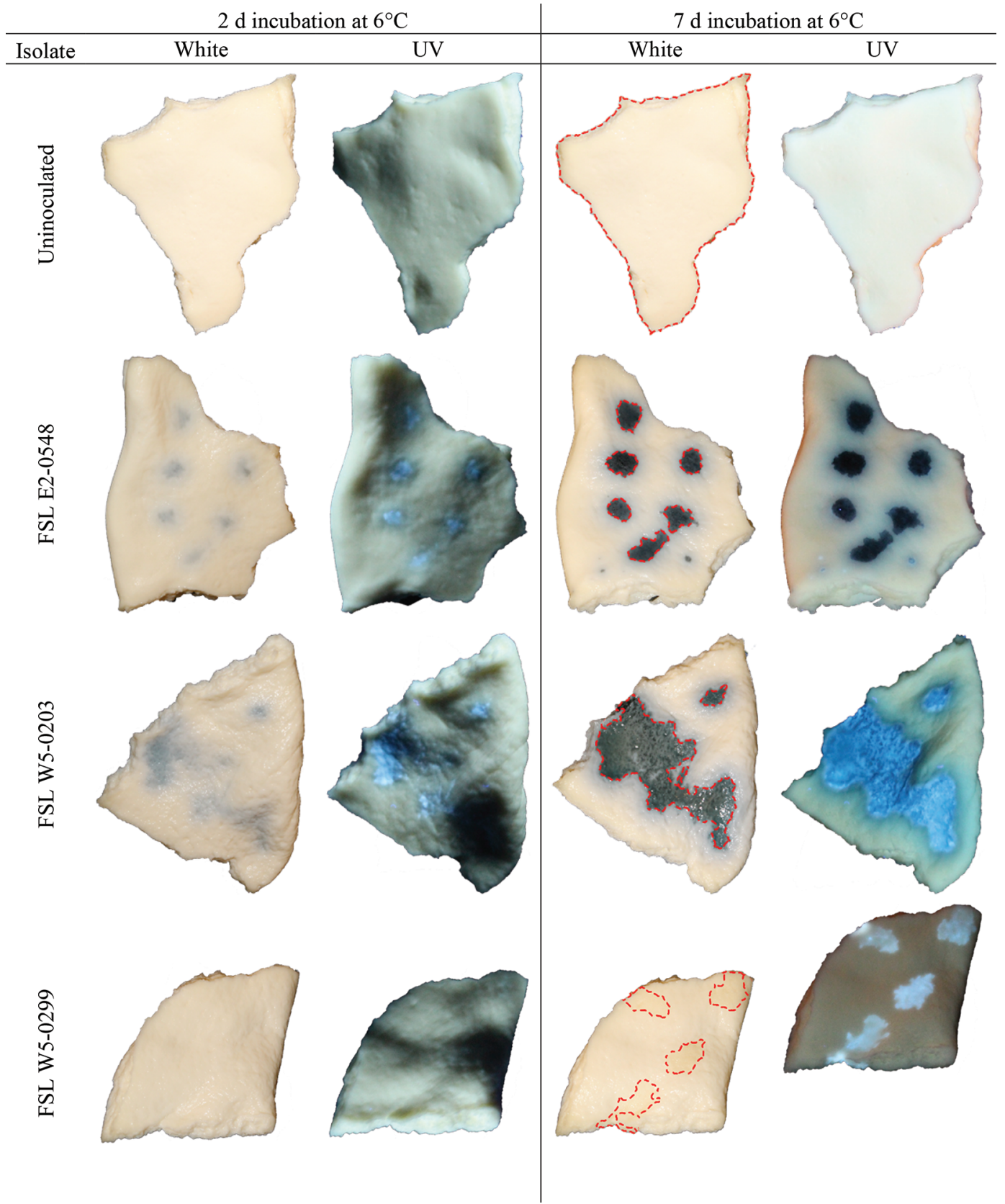

Figure 5. Photographs under white and UV light of pigment-associated Pseudomonas isolates inoculated onto mozzarella-style cheese and incubated at $6^{\circ} \mathrm{C}$ for 2 and $7 \mathrm{~d}$. Red dashed lines represent the areas in each image from $\mathrm{d} 7$ that were selected for color analysis (results in Figure 4). 
each other and from the other 7 isolates characterized here. The closest relatives of FSL W7-0098 were $P$. fragi and P. psychrophila, whereas FSL W5-0299 did not cluster with any of the Pseudomonas type strains included in the Ribosomal Database Project.

\section{ANIb Data Analysis and Clustering}

To facilitate better species classification than is possible with $16 \mathrm{~S}$ rDNA data alone, we constructed an ANIb dendrogram using WGS data (Figure 6). This dendrogram confirmed that the 6 Pseudomonas isolates previously linked to blue or gray color defects in dairy products represented 2 distinct groups. The 4 newly sequenced pigment-forming isolates that grouped with $P$. azotoformans based on $16 \mathrm{~S} \mathrm{rDNA}$ sequence data grouped with (1) the $P$. lactis type strain (the most closely related type strain based on ANIb data); (2) our control strain FSL R5-0199; (3) 2 other European strains (Ps_22 and Ps_77) previously reported to cause blue color defects in food; and (4) 2 European strains (Ps_20 and Ps_40) that had not been shown to cause blue color defects when inoculated into cheese. However, the ANIb dendrogram also indicated that this group of 10 isolates represented 2 clusters: cluster I with 4 isolates (including the P. lactis type strain DSM 29167) that were all within a pairwise ANIb of $>95.2 \%$, and cluster II with 6 isolates (all within a pairwise ANIb of $>95.0 \%$ ) that further separated into 2 distinct subclusters (IIa and IIb) of 3 isolates each. In subcluster IIa, pigmented isolate FSL E2-0548 clustered closely with Italian isolates Ps_20 and Ps_22 (unpigmented and pigmented, respectively; ANIb $>98.2 \%$ ). Notably, FSL E2-0548 and Ps_22 shared >99.7\% ANIb. In subcluster IIb, FSL W5-0203 (pigmented) and FSL R5-0199 (unpigmented) clustered closely with each other and with the pigmented isolate Ps_77 from the United Kingdom (ANIb > 98.7\%). In cluster I, the pigmented isolates FSL E2-8864 and FSL R10-2514 clustered with the unpigmented Italian isolate Ps_40 and with the unpigmented type strain P. lactis DSM 29167 (ANIb $>95.2 \%$ ). The 9 non-type strain isolates from clusters I and II had ANIb ranging from 94.2 to $95.9 \%$ with the type strain Pseudomonas lactis DSM 29167, and they therefore fell close to the lower bound of the suggested average nucleotide identity for species delineation of 95 to $96 \%$ (Richter and Rosselló-Móra, 2009). The ANIb of these 9 isolates with the type strain $P$. azotoformans (the closest match in the $16 \mathrm{~S}$ rDNA phylogenetic tree) ranged from 86.7 to $87.1 \%$, and the ANIb with the type strain $P$. fluorescens DSM 50090 ranged from 86.4 to $86.8 \%$. To determine whether or not the isolates from clusters I and II would have been identified as P. lactis had its $16 \mathrm{~S}$ rDNA sequence been included in the Ribosomal
Database Project, we constructed a second 16S rDNA maximum-likelihood tree using the 5 newly sequenced isolates from clusters I and II, the 7 Pseudomonas type strains from the Ribosomal Database Project that clustered with the newly sequenced isolates, and P. lactis DSM 29167 (Supplemental Figure S3; https://doi.org/ 10.3168/jds.2018-16192). P. lactis DSM 29167 clustered more closely with the newly sequenced isolates than the $P$. azotoformans type strain, indicating that the 5 newly sequenced isolates were more closely related to $P$. lactis than to $P$. azotoformans by $16 \mathrm{~S}$ rDNA as well. A maximum parsimony tree constructed using the pan-genome SNPs from the isolates in clusters I and II supported the groupings of the ANIb dendrogram with more resolution (Supplemental Figure S4; https://doi .org/10.3168/jds.2018-16192).

The ANIb dendrogram also confirmed that 2 additional Pseudomonas isolates previously linked to blue or gray color defects clustered closely to P. putida (consistent with the $16 \mathrm{~S}$ rDNA-based clustering). Isolates FSL R10-3386 and FSL R10-3389 clustered very closely with each other (ANIb >99.9\%) and showed an ANIb of $94.7 \%$ relative to the type strain $P$. putida NBRC 14164, which places these isolates close to the lower bound for species delineation.

\section{Genes Associated with the Gray Color Defect in Milk and the Blue Color Defect in Cheese}

We used subtractive genomics to identify genes that were specifically present in the 4 newly sequenced $P$. cf. lactis isolates (FSL E2-0548, FSL W5-0203, FSL E28864, and FSL R10-2514) and in the 2 newly sequenced P. putida isolates (FSL R10-3386 and FSL R10-3389) but missing from the other 3 newly sequenced isolates (FSL W7-0098, FSL R5-0199, and FSL W5-0299). We identified 18 genes using this approach (Supplemental Table S2; https://doi.org/10.3168/jds.2018-16192). Among these, 14 genes (including $\operatorname{trp} A, \operatorname{trp} B, \operatorname{trp} C$, $\operatorname{trp} D$, and $\operatorname{trp} F$ ) were in the same locus and were annotated with functions mainly associated with the tryptophan biosynthesis pathway. An additional 2 genes were found in this locus only among the 4 pigmented $P$. cf. lactis isolates and were not found in the 2 pigmented $P$. putida isolates. The genes in the $\operatorname{trp} A B C D F$ operon are involved in all reactions carried out in the tryptophan biosynthesis pathway, beginning from anthranilate (Figure 7). Interestingly, further analyses found that these 5 genes showed homology to 5 divergent genes, present in all genomes analyzed, where they are separated into 3 distinct loci containing $2(\operatorname{trp} A B), 1(\operatorname{trp} F)$, and $2(\operatorname{trp} C D)$ genes. Also present in the 6 pigmented isolates sequenced here and absent in the unpigmented isolates were genes encoding toxin and antitoxin pro- 
Pseudomonas resinovorans DSM 21078

Pseudomonas otitidis DSM 17224

Pseudomonas mendocina NBRC 14162

Pseudomonas alcaligenes NBRC 14159

Pseudomonas thermotolerans DSM 14292

Pseudomonas azotifigens DSM 17556

Pseudomonas aeruginosa DSM 50071

Pseudomonas flexibilis ATCC 29606

Pseudomonas oryzihabitans NBRC 102199

Pseudomonas psychrotolerans DSM 15758

Pseudomonas stutzeri ATCC 17588 LMG 11199

Pseudomonas stutzeri ATCC 14405 CCUG 16156

Pseudomonas balearica DSM 6083

Pseudomonas xanthomarina DSM 18231

Pseudomonas syringae DSM 10604

Pseudomonas congelans DSM 14939

Pseudomonas viridiflava DSM 6694

Pseudomonas cichorii ATCC 10857

Pseudomonas lutea DSM 17257

Pseudomonas abietaniphila ATCC 700689

FSL W5-0299

Pseudomonas lini DSM 16768

Pseudomonas chlororaphis ATCC 13985

Pseudomonas corrugata DSM 7228

Pseudomonas veronii DSM 11331

Pseudomonas fluorescens DSM 50090

Pseudomonas cedrina DSM 17516

Pseudomonas azotoformans DSM 18862

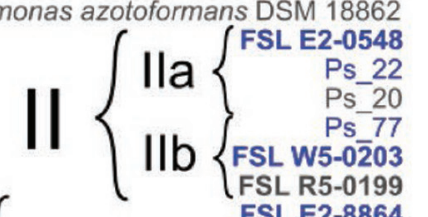

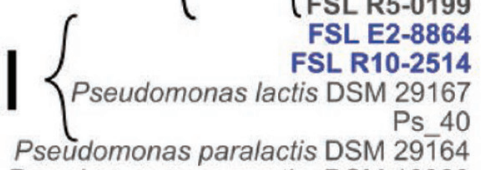

Pseudomonas paralactis DSM 29164
Pseudomonas synxantha DSM 18928

Pseudomonas libanensis DSM 17149

Pseudomonas orientalis DSM 17489

Pseudomonas trivialis DSM 14937

Pseudomonas poae DSM 14936

Pseudomonas gessardii DSM 17152

Pseudomonas rhizosphaerae DSM 16299

Pseudomonas psychrophila DSM 17535

Pseudomonas deceptionensis DSM 26521

FSL W7-0098

Pseudomonas taetrolens DSM 21104

Pseudomonas helleri DSM 29165

Pseudomonas weihenstephanensis DSM 29166

Pseudomonas lundensis DSM 6252

FSL R10-3389

FSL R10-3386

Pseudomonas putida NBRC 14164

Pseudomonas monteilii NBRC 103158 DSM 14164 Pseudomonas plecoglossicida NBRC 103162 DSM 15088

Pseudomonas mosselii DSM 17497

Pseudomonas taiwanensis DSM 21245

Pseudomonas parafulva NBRC 16636 DSM 17004

Pseudomonas fulva NBRC 16636 DSM 17004

Pseudomonas cremoricolorata DSM 17059

Pseudomonas japonica NBRC 103040

Pseudomonas vranovensis DSM 16006

Pseudomonas bauzanensis DSM 22558

Pseudomonas caeni DSM 24390

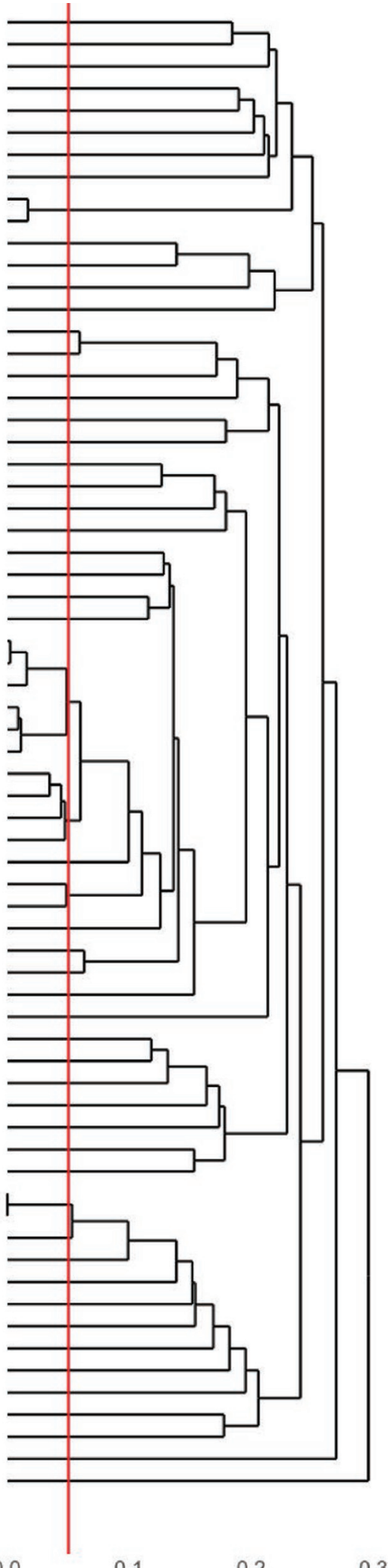
0.0
0.1
0.2
0.3

Figure 6. Dendrogram of the whole-genome-sequenced Pseudomonas isolates generated using the whole-genome average nucleotide identity by basic local alignment search tool (ANIb) distance matrix. Newly sequenced isolates are in bold text, and isolates known to produce a gray or blue pigment are in blue text. The red vertical line represents $95 \%$ ANIb, and the numbers along the horizontal axis represent pairwise nucleotide distance. Clusters and subclusters identified in the Pseudonomas fluorescens group are denoted using curly braces. 


\begin{tabular}{|c|c|c|}
\hline Locus & $\begin{array}{l}\text { Gene } \\
\text { name }\end{array}$ & Annotation \\
\hline FSL_E2-0548_02093 & wbpA & UDP-N-acetyl-D-glucosamine 6-dehydrogenase \\
\hline FSL_E2-0548_02094 & $\operatorname{trpD}$ & Anthranilate phosphoribosyltransferase \\
\hline FSL_E2-0548_02095 & $\operatorname{trpF}$ & $\mathrm{N}$-(5'-phosphoribosyl)anthranilate isomerase \\
\hline FSL_E2-0548_02096 & $\operatorname{trpA}$ & Tryptophan synthase alpha chain \\
\hline FSL_E2-0548_02097 & $\operatorname{trp} B$ & Tryptophan synthase beta chain \\
\hline FSL_E2-0548_02098 & group_6931 & hypothetical protein \\
\hline FSL_E2-0548_02099 & gfo & Glucose--fructose oxidoreductase \\
\hline FSL_E2-0548_02100 & yjmc & putative oxidoreductase YjmC \\
\hline FSL_E2-0548_02101 & lysK & [LysW]-lysine hydrolase \\
\hline FSL_E2-0548_02102 & IOIE & Inosose dehydratase \\
\hline FSL_E2-0548_02103 & $w b p E$ & $\begin{array}{l}\text { UDP-2-acetamido-2-deoxy-3-oxo-D-glucuronate } \\
\text { aminotransferase }\end{array}$ \\
\hline FSL_E2-0548_02104 & $w b p E$ & $\begin{array}{l}\text { UDP-2-acetamido-2-deoxy-3-oxo-D-glucuronate } \\
\text { aminotransferase }\end{array}$ \\
\hline FSL_E2-0548_02106 & $\operatorname{trpc}$ & Indole-3-glycerol phosphate synthase \\
\hline FSL_E2-0548_02108 & $r h t B$ & Homoserine/homoserine lactone efflux protein \\
\hline
\end{tabular}

B chorismate L-glutamine

TrpE $\longrightarrow$ anthranilate

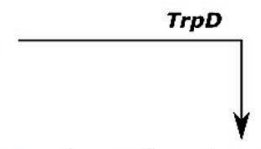

5-phosphoribosyl-anthranilate

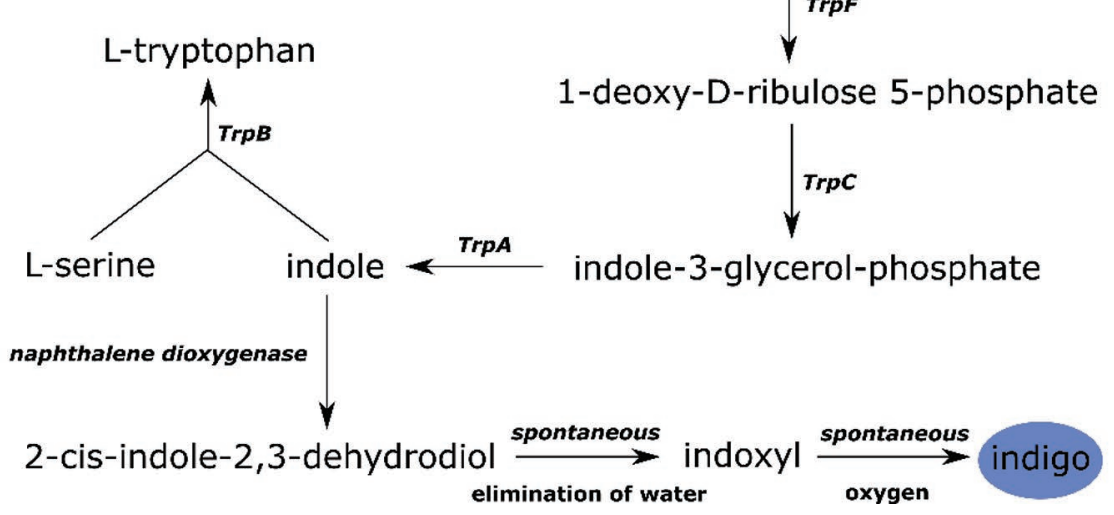

Figure 7. The putative roles of accessory tryptophan biosynthesis genes found in Pseudomonas isolate FSL E2-0548 in the tryptophan and indigo biosynthetic pathways. All pigmented Pseudomonas isolates possessed these accessory genes. 
teins $(C h p B-C h p S)$ and an L-homoserine lactone exporter $(r h t B)$.

\section{Evolution of Genes Involved Both in Tryptophan Biosynthesis and Indigo Biosynthesis}

As was indicated by our own data from the preceding section and by a previous study on 2 Pseudomonas isolates linked to a blue color defect in food products (Andreani et al., 2015a), there appears to be a linkage between blue or gray color phenotypes and the presence of genes encoding part of the tryptophan biosynthesis pathway. The 8 pigmented isolates, FSL E2-8864, FSL R10-2514, FSL E2-0548, FSL W5-0203, FSL R10-3386, FSL R10-3389, Ps_22, and Ps_77, harbored 2 copies of $\operatorname{trp} A, \operatorname{trp} B, \operatorname{trp} C, \operatorname{trp} D$, and $\operatorname{trpF}$; the other 5 isolates, which showed no color defect, harbored 1 copy of each gene. Maximum-likelihood trees created from multiple alignments of the predicted amino acid sequences of the genes showed that 1 of the 2 copies of the $\operatorname{trp} A$, $\operatorname{trp} B, \operatorname{trp} C, \operatorname{trp} D$, and $\operatorname{trp} F$ genes from all 8 of the pigmented isolates clustered together (Figures $8 \mathrm{a}-\mathrm{e}$ ). To differentiate the 2 distinct copies of these genes, we designated the $\operatorname{trp}$ genes found in all isolates as $\operatorname{trp} A-1$ through $\operatorname{trp} F-1$, and we designated the $\operatorname{trp}$ genes found only in the pigmented isolates as $\operatorname{trp} A-2$ through $\operatorname{trp} F$ 2 . We did not include Ps_22 in the $\operatorname{trp} B$ tree because its $\operatorname{trp} B$-2 sequence was incompletely sequenced in the previously published Ps_22 genome (Andreani et al., 2015a); $\operatorname{trp} A-2, \operatorname{trp} C-2, \operatorname{trp} D-2$, and $\operatorname{trpF-2}$ for Ps_22 clustered with the remaining 7 pigmented isolates as detailed below. With this exception, in all 5 gene trees, 1 copy of each gene consistently grouped the 8 pigmented isolates together in a cluster consisting of 1 branch $(\operatorname{trp} D$-2) or 2 branches $(\operatorname{trp} A$-2, $\operatorname{trp} B$-2, $\operatorname{trp} C$-2, and $\operatorname{trp} F$-2). In cases of 2 branches, 1 branch consisted of $P$. cf. lactis isolates FSL E2-8864, FSL R10-2514, FSL E2-0548, FSL W5-0203, Ps_22, and Ps_77, and 1 branch consisted of $P$. putida isolates FSL R10-3386 and FSL R10-3389. For $\operatorname{trp} A-1, \operatorname{trp} B-1, \operatorname{trp} C-1, \operatorname{trp} D-1$, and $\operatorname{trpF-1}$, all of which were found in all 13 isolates, the genes for pigmented and unpigmented isolates did not represent separate clusters.

\section{Hidden Markov Models}

We ran hidden Markov models for 13 protein families or domains on the translated protein-coding sequences from the whole genome sequences of each of the 9 newly sequenced Pseudomonas isolates. We found significant evidence using 6 of the models for the presence of protein families or domains associated with pyocyanin, tryptophan, and plastocyanin copper-binding proteins in all 9 genomes tested, whether from pigmented or unpigmented isolates. We found a lack of evidence for the presence of 5 other protein families or domains that were associated with pyoverdine, indigoidine, or violacein biosynthesis in all 9 tested genomes. The remaining 2 models, a protein family encoding the N-terminal of a protein putatively involved in pyoverdine biosynthesis $(\mathrm{HpaB})$ and a protein domain encoding membranebound blue copper proteins $(\operatorname{Cop} C)$, were present in 1 or more predicted proteins for 7 of the 9 isolates $(P$. putida isolates FSL R10-3386 and FSL R10-3389 were missing these predicted proteins). Overall, the hidden Markov models failed to detect any protein families or domains associated with pigment production that were present only in the pigmented isolates and absent in the unpigmented isolates. Corroborating the results found in the comparative genomics analysis, 2 copies of predicted tryptophan biosynthesis domain Trp_syntA were found in pigmented isolates, whereas only 1 copy was found in unpigmented isolates.

\section{DISCUSSION}

\section{A Diversity of Pseudomonas Can Cause Gray Defects in Fluid Milk}

The Pseudomonas spp. that can cause either a gray color defect in milk or a blue color defect in cheese can represent at least 2 very distinct taxonomic groups, including (1) a group that we can currently classify as $P$. cf. lactis [we used the designation cf. (confer) because these isolates are closely related to the $P$. lactis type strain but border the currently accepted cutoff for speciation by whole genome average nucleotide identity] and (2) a group that we can likely classify as $P$. putida. These findings illustrate the improved ability of taxonomic and species classification when using WGS data compared with using $16 \mathrm{~S}$ rDNA data, which have not allowed for reliable classification of Pseudomonas isolates in several studies (Gomila et al., 2015; GarridoSanz et al., 2016; Peix et al., 2018).

Members of the group of 6 pigmented $P$. cf. lactis isolates sequenced previously and in this work had been reported as $P$. azotoformans (Evanowski et al., 2017), P. fluorescens biovar IV (Martin et al., 2011), and P. fluorescens (Andreani et al., 2015a). Based on ANIb analysis of WGS data, our data clearly support the concept that these isolates form a single group of related strains that likely represent a single species or perhaps 2 closely related species. Specifically, this group includes 9 total isolates along with the P. lactis type strain, with overall pairwise ANIb of $\geq 94 \%$ (slightly below the species cutoff of 95\%). Separation of this overall group into 2 clusters yielded cluster I with 3 isolates (along with the P. lactis type strain) and cluster II 
a)

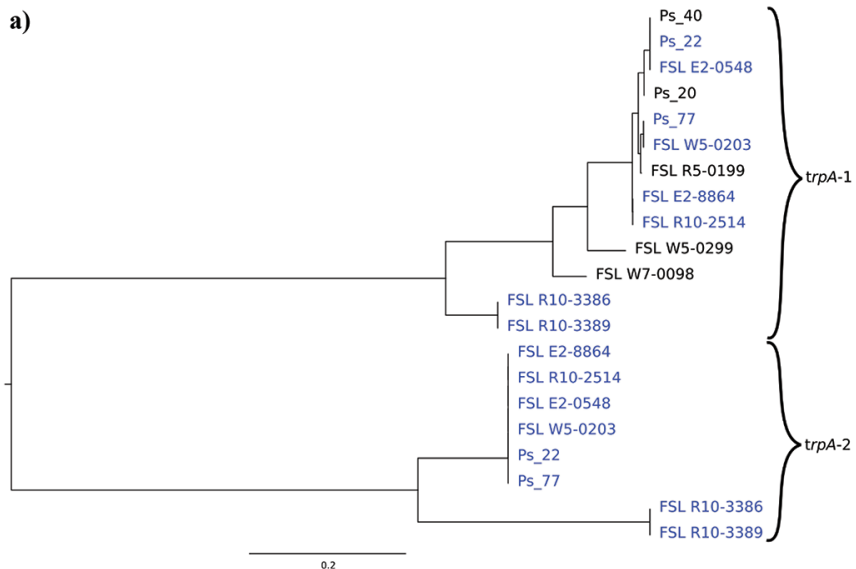

b)

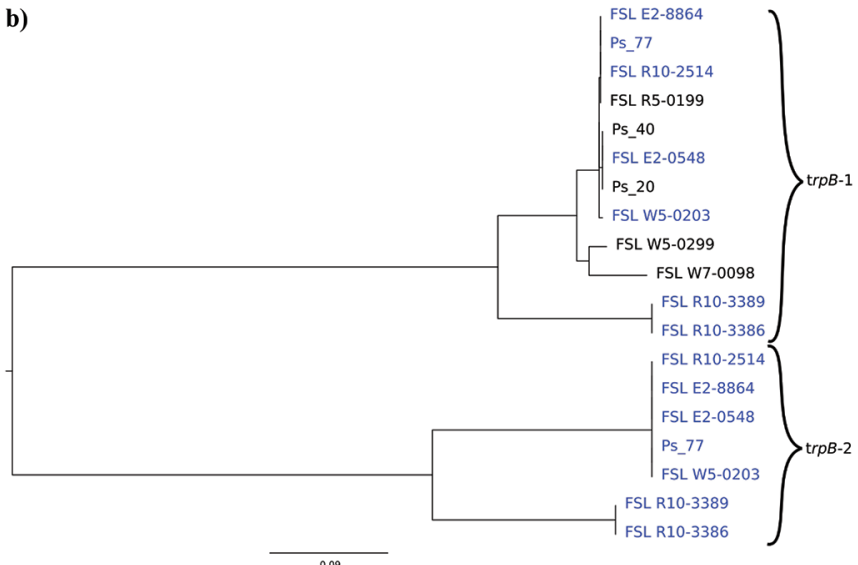

c)

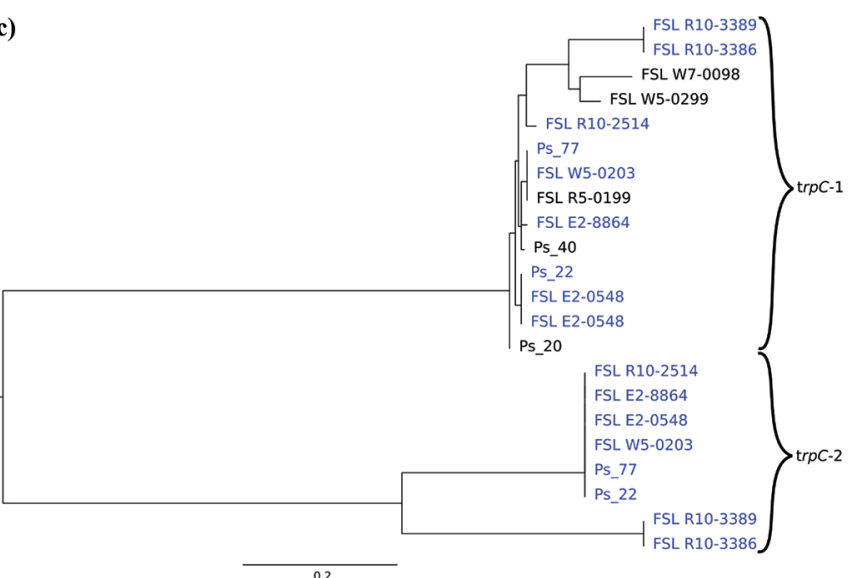

d)

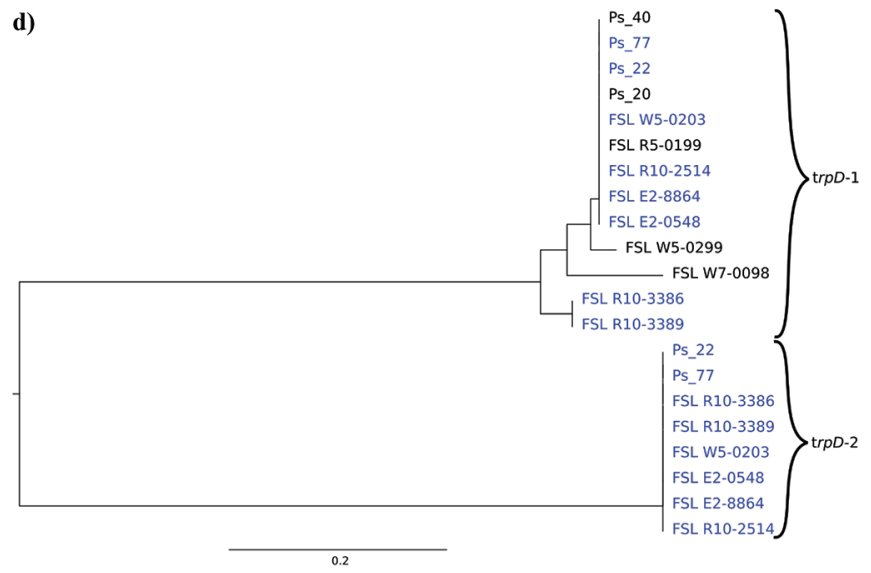

e)

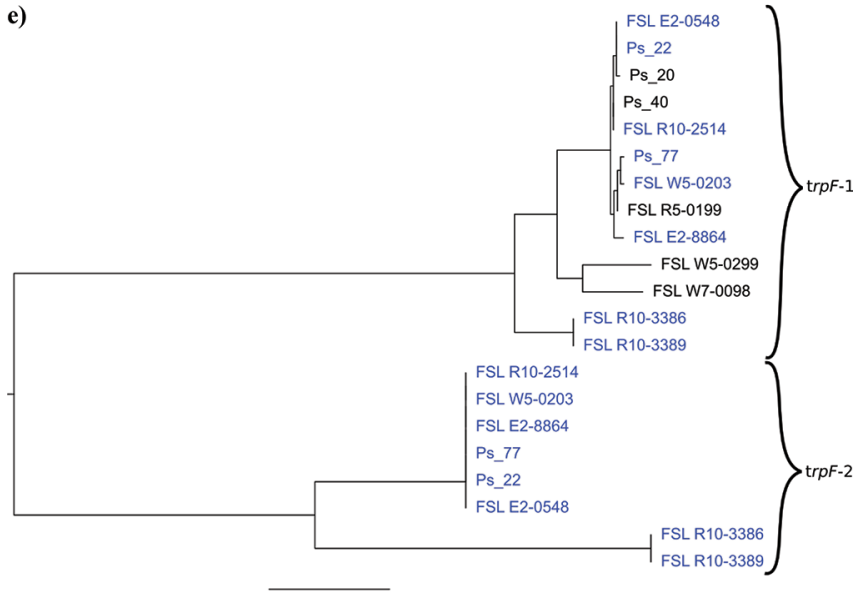

Figure 8. Phylogeny of proteins encoded by (a) $\operatorname{trp} A$, (b) $\operatorname{trp} B$, (c) $\operatorname{trp} C$, (d) $\operatorname{trp} D$, and (e) $\operatorname{trp} F$ involved in tryptophan biosynthesis from all newly sequenced Pseudomonas isolates and in previously sequenced isolates Ps_20, Ps_22, Ps_40, and Ps_77. The maximum-likelihood trees are drawn to scale, with branch lengths and the scale bar measured in the number of AA substitutions per site. Isolate names in blue text have been shown to produce gray or blue pigment. Curly braces have been used to identify phylogenetic clusters.

with 6 isolates. Each cluster had pairwise ANIb values of $>95.0 \%$. Although one could argue that cluster II represents a new species (when applying the previously proposed $95 \%$ cutoff as a strict rule), we elected to classify all 9 isolates as a single species ( $P$. cf. lactis), at least for the time being, allowing for collection and characterization of additional isolates and generation of further data before making a taxonomic proposal that contributes to the already rapidly expanding number of new Pseudomonas spp. Previous studies that classified isolates in this group to the species level did not use WGS data for classification. For example, our group 
reported that, based on $16 \mathrm{~S}$ rDNA data, a Pseudomonas isolate that caused a gray defect in milk (FSL E2-0548) could be classified as P. azotoformans (Evanowski et al., 2017). This finding is consistent with the $16 \mathrm{~S}$ data analysis we have reported here, but the ANIb data generated for this study showed that this isolate had only $86.8 \%$ similarity to the P. azotoformans type strain and $94.2 \%$ similarity to the $P$. lactis type strain and classified it into P. lactis cluster II. Similarly, our group used $16 \mathrm{~S}$ rDNA sequence data to classify a single isolate obtained from blue-pigmented cheese (FSL W50203) as P. fluorescens biovar IV (Martin et al., 2011), whereas it showed only $86.9 \%$ ANIb similarity to the $P$. fluorescens type strain. An Italian group also previously reported whole genome sequences for 2 isolates (Ps_22 and Ps_77) that caused blue color defects in cheese as well as 2 closely related isolates (Ps_20 and Ps_40) that did not cause this type of defect (Andreani et al., 2015a). These 4 isolates were classified into the $P$. fluorescens group without reporting a more specific species classification. The WGS data analyses we have reported here support the idea that $P$. lactis, which was first reported in 2017 (von Neubeck et al., 2017), represents a species that includes several distinct strains that can cause blue or gray color defects in dairy products. Several Pseudomonas species have been linked to the spoilage of dairy products, but identification of $P$. lactis isolates in products or processing environments may indicate an enhanced risk of color defects. It is, however, important to note that many currently used species classification approaches for Pseudomonas may not correctly identify a given isolate as $P$. lactis (e.g., as of December 5, 2018, a P. lactis 16S sequence was not included in the Ribosomal Database Project) and may misidentify $P$. lactis isolates as closely related species including, but not limited to, P. azotoformans.

Interestingly, we also found 2 isolates that caused a color defect when inoculated into milk, did not cause a color defect on PDA, are not closely related to $P$. lactis, and likely represent the species $P$. putida. These 2 pigmented isolates were very closely related to each other and showed an ANIb similarity of $94.7 \%$ to the $P$. putida type strain. Importantly, this clarifies that Pseudomonas strains closely related to $P$. putida are also able to cause blue or gray color defects in dairy products. Interestingly, these 2 historical isolates were described by Hammer (1914), who investigated an "outbreak" of blue milk in Iowa and originally identified these isolates as Bacillus cyanogenes. Bacillus cyanogenes was later reclassified as Pseudomonas syncyanea or P. cyanogenes (Hammer, 1957), but neither of these species is present in the most recent edition of Bergey's Manual (Palleroni, 2015). In addition, the 2 isolates characterized here were submitted to the USDA Agri- cultural Research Service Culture Collection by B. W. Hammer under the names $P$. mildenbergii and P. cyanogena, but these species do not appear in the current Bergey's Manual either (Haynes, 1961; Palleroni, 2015). The collection currently lists these isolates as $P$. putida (USDA Agricultural Research Service, 2018), and we verified that this classification is accurate by WGS. Overall, our findings indicate that a larger diversity of Pseudomonas species than is generally assumed can cause color defects in dairy products, and they illustrate the value of accessible long-term strain collection maintenance.

\section{Some Pseudomonas Strains Can Cause Both Gray Color Defects in Milk and Blue Color Defects on Cheese}

Through inoculation and WGS experiments involving isolate FSL E2-0548 from fluid milk and isolate FSL W5-0203 from cheese, we demonstrated that these 2 isolates are both closely related to each other and are able to produce color defects in dairy products other than those from which they originated (i.e., FSL E2-0548 produced blue pigment on cheese and FSL W5-0203 produced gray pigment in milk). This inoculation experiment, along with the $>99.7 \%$ ANIb shared between pigment-producing isolates FSL E2-0548 (milk) and Ps_22 (cheese), strongly suggests that many blue- or gray-pigment-producing Pseudomonas associated with dairy foods should be able to produce pigment defects in multiple dairy products. The reason why fluid milk appears gray when contaminated with these Pseudomonas whereas cheese turns blue is relatively unexplored, but is supported by historical reports. A gray color in milk similar to what we observed was reported by Duckwall (1905) and by Hammer (1914) when samples of sterilized milk were inoculated with their respective isolates of blue-pigmented Pseudomonas. Both authors observed blue pigment in their Pseudomonas-inoculated sterilized milk when the milk was acidified - chemically in the case of Duckwall, and when cocultured with lactic acid bacteria in the case of Hammer. The efficacy of both approaches to acidification suggests that $\mathrm{pH}$, rather than other fermentation-related changes in the milk, determines the ultimate appearance of the pigment. It is likely that modern high-temperature shorttime pasteurized milk appears gray when contaminated due to its near-neutral $\mathrm{pH}$ and its low prevalence of lactic acid bacteria that reach spoilage levels (i.e., reduce the $\mathrm{pH}$ ) over shelf life (Reichler et al., 2018). By extension, it could be surmised that blue, rather than gray, pigment was produced on mozzarella cheese by these Pseudomonas because of the acidification of the cheese during production, having much the same ef- 
fect as has been reported in fluid milk. Other potential explanations for the differences in the color produced in milk and on cheese are that multiple pigments are produced, that the pigment is altered after its synthesis, and that the perceived color of the pigment or pigments depends on environmental conditions other than $\mathrm{pH}$ (e.g., nutrients, oxygen).

Importantly for the dairy industry, isolates associated with blue or gray pigment defects have been isolated from dairy and other food products both in the United States and throughout the European Union, including Great Britain, Spain, Germany, and Italy (Andreani et al., 2015b; del Olmo et al., 2018). The occurrence of these strains in global regions that are noteworthy, even famous, for their dairy products leaves open the risk of future high-profile mass spoilage events similar to what occurred in Italy in 2010 (Greenhalgh, 2010). In the United States, we have heard anecdotally that periods of contamination at dairy processing facilities have led to increased levels of consumer complaints due to gray pigment defects in fluid milk. Because even a single spectacularly spoiled unit of product has the potential to cause damage to a brand if disseminated in photographs or videos via social media, it is imperative that producers of fresh, uncultured milk and cheese products take the necessary precautions to prevent postpasteurization contamination with pigment-producing Pseudomonas. Such precautions include the implementation or improvement of sanitation standard operating procedures, preventive maintenance protocols, and employee training.

It is important to note that the gray pigmentation in milk does not result from an unrealistically large population of Pseudomonas. In our study, the gray color defect was observed in milk that reached approximately 8 $\log \mathrm{cfu} / \mathrm{mL}$. This level of Pseudomonas can realistically be achieved in commercial fluid milk over $21 \mathrm{~d}$ of storage at refrigeration temperatures, particularly under conditions of slight temperature abuse. For example, Reichler et al. (2018) observed that milk contaminated with Pseudomonas at the processing facility showed an average SPC of $8.23 \mathrm{log} \mathrm{cfu} / \mathrm{mL}$ after $21 \mathrm{~d}$ of storage at $6^{\circ} \mathrm{C}$.

\section{Tryptophan and Indigo Biosynthesis Genes Are Likely Associated with the Formation of Color Defects in Foods by Pseudomonas spp. Strains}

Our comparative genomics analysis identified only 18 genes that were present in all 6 genomes associated with the gray color defect in fluid milk and absent in all 3 genomes not associated with this color defect. Among those genes, 14 were clustered in a single locus that had been previously found in a European Pseudomonas isolate that produced a blue color defect in mozzarella cheese (Andreani et al., 2015a). Interestingly, the 16 genes of this locus reported by Andreani et al. were present in all of our newly sequenced $P$. cf. lactis isolates, but only 14 genes were detected in the newly sequenced $P$. putida isolates. This may be because of extensive evolutionary divergence between the $P$. cf. lactis isolates and the $P$. putida isolates, or it may represent either the gain of these 2 genes by the ancestor of the $P$. cf. lactis isolates or the loss of them by the ancestor of the P. putida isolates. This locus includes 5 genes that encode for proteins involved in the tryptophan biosynthesis pathway. All Pseudomonas shown to produce the gray color defect in fluid milk or the blue color defect in cheese present 2 copies of these 5 genes, whereas Pseudomonas that do not produce either of these color defects harbor a single copy of the genes. Interestingly, our data are consistent with the previous suggestion (Andreani et al., 2015a) that this second tryptophan operon copy may have been transferred horizontally across a diverse population of Pseudomonas, resulting in its presence in 2 distinct Pseudomonas groups. The horizontal gene transfer hypothesis is supported by the observation that the genes in the second copy of this operon, which is present only among pigmented isolates, are phylogenetically closely related and show much lower nucleotide diversity than the copies present in all Pseudomonas. Interestingly, indole is produced during tryptophan biosynthesis, which can then be converted into indigo or an indigo-like molecule (which typically exhibit a color that is close to blue) in a reaction that is oxygen-dependent (Ensley et al., 1983). The oxygen dependence of the indigo production, combined with the observation that the gray color defect was only observed in half-empty containers of fluid milk (which leads to increased oxygen exposure) and previous reports that the defect often showed up as a ring on the milk surface, makes it highly plausible that this indigo reaction is responsible for the production of the gray milk defect. Functionally, an additional tryptophan operon may be regulated in a way that facilitates tryptophan synthesis in refrigerated fluid milk, whereas the broadly distributed operon is not expressed in milk. Further experimental work is needed to test this hypothesis. Another alternative hypothesis that could be tested experimentally is that the second horizontally transferred trp operon encodes proteins with enhanced activity in a dairy environment or at low temperatures. In addition to the 5 genes encoding enzymes involved in tryptophan biosynthesis, the locus specifically present in the pigmented isolates also included the gene $\operatorname{rht} B$, which encodes for an L-homoserine and L-homoserine lactone exporter. Because L-homoserine lactone has been shown to function as a quorum-sensing molecule 
(i.e., its presence in the environment signals that a high population density has been reached; Zakataeva et al., 1999; Dunstall et al., 2005), it is possible that it serves as a signal for biosynthesis of the pigment as the cell density reaches a minimum threshold. No other gene within the locus appears to be involved in gene regulation.

We also tested the hypothesis that all strains that produce gray milk or blue cheese could express specific blue or gray pigments other than indigo. This hypothesis was considered appropriate, because several other blue or violet pigmented molecules have been described in Pseudomonas (e.g., pyocyanin, indigoidine relatives, hydroxamic acids, blue copper proteins; Cha and Cooksey, 1991; Budzikiewicz, 2010; Palleroni, 2015). Although hidden Markov model searches identified several genes that were predicted to encode pathways for potential blue or gray pigments, none of these was specifically associated with the strains that caused the gray milk pigment defects. For example, although genes involved in pyoverdine biosynthesis were found among the genomes of the pigmented isolates, these genes were also found among the genomes of the unpigmented isolates. Of note, however, no models existed for hydroxamic acid or hydroxamate biosynthesis, and hydroxamates are known for their $\mathrm{pH}$-dependent formation of reddish-brown to purple complexes with iron (Neilands, 1967). Hydroxamates have been reported both in P. fluorescens group isolates (Shirahata et al., 1970; Philson and Llinás, 1982) and in P. mildenbergii (Hulcher, 1982), a currently invalid species that once included our 2 historical $P$. putida isolates.

\section{Closely Related Pseudomonas cf. lactis Isolates that Cause Near-Identical Color Defects on Cheese and in Milk Differ in Their Color Phenotypes on PDA}

Our analyses identified the duplication of a tryptophan biosynthesis operon as a likely mechanism linked to the gray milk and blue cheese defects, but we also found that the Pseudomonas isolates we tested caused several different color phenotypes when grown on PDA at $6^{\circ} \mathrm{C}$, even though they showed the same phenotype when inoculated in milk incubated at $21^{\circ} \mathrm{C}$. For example, isolate FSL E2-0548, which originated from gray milk but also produced a blue pigment on cheese, produced a reddish-brown pigment on PDA after $5 \mathrm{~d}$ of incubation at $6^{\circ} \mathrm{C}$. On the other hand, isolate FSL W5-0203 produced a blue pigment on both cheese and PDA. The difference in pigmentation on PDA compared with cheese and milk, as well as the diversity of color phenotypes on PDA, may be attributed to several, not necessarily mutually exclusive, causes, including (1) production of additional or different pigments on
PDA; (2) different color appearance of a given pigment on cheese and PDA due to compositional differences (e.g., pH); (3) production of a supplemental factor that either stabilizes or degrades the pigment on PDA; or (4) strains differing in their ability to alter their growth environment in different media (e.g., acid production from glucose), resulting in different visible colors. Some of these hypotheses have been raised by previous papers (Duckwall, 1905; Hammer, 1914; Andreani et al., 2015b) that reported expression of gray and brown pigments by blue-pigment-producing Pseudomonas strains grown on agar plates. These authors suggested that these observations could be due to additional pigments being produced or to the blue pigment being altered upon exposure to oxygen. In our experiments, the hypothesis that oxygen exposure is responsible for the alteration of the pigment from blue to brown did not seem relevant, because both cheese (where a blue color was observed) and PDA (where a gray color was observed) were held without immersion in brine or other liquid in unsealed Petri dishes with ample oxygen exposure. Additionally, other isolates have been reported to change color from blue to gray or brown over the course of incubation (Andreani et al., 2015b), but this was not the case for FSL E2-0548, which never exhibited blue pigment on PDA media, or for FSL W5-0203, which has been observed to produce only a stable blue pigment (Martin et al., 2011).

The hypothesis that the color-producing Pseudomonas strains studied here produce additional pigments on PDA seemed initially attractive, but hidden Markov model searches did not identify any pigment production pathways that could explain our findings. However, the available hidden Markov models are unlikely to identify all pigment pathways that may be responsible for the different color phenotypes observed. The hypothesis that a given pigment may show different color on cheese and PDA is supported by historical publications that indicate that lower $\mathrm{pH}$ (as found in cheese) has been reported to enhance blue pigmentation in dairy spoilage Pseudomonas (Hammer, 1914; Mildenberg, 1922). With respect to the hypothesis that a supplemental factor could stabilize or degrade color formed in dairy or PDA, it is possible that the citric acid used to acidify the milk for cheesemaking exerts a protective role on the blue pigment, because citric acid is known to inhibit enzymatic browning in fruits and vegetables and to chelate metal ions that could directly catalyze oxidative reactions of pigments or act as enzymatic cofactors (Lindsay, 1996; Whitaker, 1996; Brady, 2013). Interestingly, historical reports also suggested that increasing amounts of glucose (which is a major ingredient of PDA) create more intense pigments without acid production in blue-pigment-producing 
Pseudomonas (Duckwall, 1905; Hammer, 1914); however, mechanisms of this color intensification have not been reported or explored and could be multifold. UV fluorescence disappeared from the cheese inoculated with FSL E2-0548 between d 2 and 7 of incubation, whereas fluorescence remained after $7 \mathrm{~d}$ for the cheese inoculated with FSL W5-0203. This difference suggests that the blue pigment and the fluorescent pigment are mutually exclusive, and that some factor differs between these 2 isolates that may explain the colors of the isolates on PDA. Overall, our findings, as well as the previous literature referenced in this section, clearly indicate that production of pigments and color by Pseudomonas present in dairy products is complex and may involve several pathways that directly and indirectly relate to color formation. Although control of color defects will not necessarily require future research into relevant pigment formation pathways, this type of future research will likely provide insights that could be used to help enhance rapid detection of color-producing Pseudomonas (e.g., through molecular methods targeting appropriate genes or through media that facilitate and enhance color production) or prevent color formation in dairy products (e.g., through inhibitors of pigment production pathways).

\section{CONCLUSIONS}

Our findings, combined with those of other studies conducted mainly in Italy, show that a range of Pseudomonas strains are likely able to cause visual color defects in dairy products that could result in their broadcast via social media. One may argue that control of these types of visually apparent spoilage issues is simple and only requires prevention of any post-kill step recontamination, but this may not be practically achievable considering that, in theory, contamination of a large volume of milk (e.g., 1 US gallon, slightly less than $4 \mathrm{~L}$ ) with a single viable cell of Pseudomonas is sufficient to allow for the gray color defect to occur over a 21-d shelf life under slightly abusive conditions. Therefore, further development of detection methods to rapidly identify pigmented Pseudomonas that could cause color defects is important. In this context, our finding that certain $P$. putida strains cause color defects in milk without showing coloration on plating media (e.g., PDA) is highly relevant, because classical microbiological methods may not easily detect these strains. Fortunately, our data provide molecular targets, such as the accessory tryptophan biosynthesis genes (e.g., $\operatorname{trp} D$ ), that could be used to develop initial molecular tests for the identification of color-producing Pseudomonas that belong to both P. putida and P. cf. lactis.

\section{ACKNOWLEDGMENTS}

The work presented here would not have been possible without the continued support of the Milk Quality Improvement Program by the New York State Dairy Promotion Advisory Board (Albany, NY; OSP \#83562) through the New York State Department of Agriculture and Markets (Albany, NY). We thank the USDA Agricultural Research Service Culture Collection for their provision of bacterial isolates for this study and for their long-term maintenance of a collection that contains resources critical to studies such as these. We also acknowledge USDA NIFA Hatch Appropriations (project PEN04646, accession 1015787). We thank Sean Guo at Cornell University for his assistance with the preparation of DNA for WGS, as well as the US dairy processors who brought this defect to our attention and provided us with the samples of defective product from which we obtained our pigmented isolates.

\section{REFERENCES}

Andreani, N. A. 2016. Into the blue: Spoilage phenotypes of Pseudomonas fluorescens in food matrices. PhD thesis. Università Degli Studi Di Padova, Padova, Italy.

Andreani, N. A., L. Carraro, M. E. Martino, M. Fondi, L. Fasolato, G. Miotto, M. Magro, F. Vianello, and B. Cardazzo. 2015a. A genomic and transcriptomic approach to investigate the blue pigment phenotype in Pseudomonas fluorescens. Int. J. Food Microbiol. 213:88-98. https://doi.org/10.1016/j.ijfoodmicro.2015.05.024.

Andreani, N. A., M. E. Martino, L. Fasolato, L. Carraro, F. Montemurro, R. Mioni, P. Bordin, and B. Cardazzo. 2015b. Reprint of 'tracking the blue: A MLST approach to characterise the Pseudomonas fluorescens group'. Food Microbiol. 45(A):148-158. https:// doi.org/10.1016/j.fm.2014.11.011.

Bankevich, A., S. Nurk, D. Antipov, A. A. Gurevich, M. Dvorkin, A. S. Kulikov, V. M. Lesin, S. I. Nikolenko, S. Pham, A. D. Prjibelski, A. V. Pyshkin, A. V. Sirotkin, N. Vyahhi, G. Tesler, M. A. Alekseyev, and P. A. Pevzner. 2012. SPAdes: a new genome assembly algorithm and its applications to single-cell sequencing. J. Comput. Biol. 19:455-477. https://doi.org/10.1089/cmb.2012.0021.

Beyerinck, M. W. 1892. La biologie d'une bactérie pigmentaire. Archives Néerlandaises Sciences Exactes Naturelles 25:227-280.

Bolger, A. M., M. Lohse, and B. Usadel. 2014. Trimmomatic: A flexible trimmer for Illumina sequence data. Bioinformatics 30:21142120. https://doi.org/10.1093/bioinformatics/btu170.

Boor, K. J., M. Wiedmann, S. Murphy, and S. Alcaine. 2017. A 100year review: microbiology and safety of milk handling. J. Dairy Sci. 100:9933-9951. https://doi.org/10.3168/jds.2017-12969.

Borowski. 1788. Bericht und gutachten über das blauwerden der milch in der molferen des königl. Amts frauendorf ben frankfurt an der ober. Neues Magazin für die Gerichtliche Arzneikunde und Medizinische Polizei 2:103-133.

Brady, J. W. 2013. Food additives. Pages 561-572 in Introductory Food Chemistry. Comstock Publishing Associates, Ithaca, NY.

Budzikiewicz, H. 2010. Microbial siderophores. Pages 1-75 in Progress in the Chemistry of Organic Natural Products. Vol. 92. A. D. Kinghorn, H. Falk, and J. Kobayashi, ed. Springer, Vienna, Austria. https://doi.org/10.1007/978-3-211-99661-4_1.

Cantoni, C., and C. Bersani. 2010. Mozzarelle blu: cause ed ipotesi. Ind. Aliment. 49:27-30.

Caputo, L., L. Quintieri, D. M. Bianchi, L. Decastelli, L. Monaci, A. Visconti, and F. Baruzzi. 2015. Pepsin-digested bovine lactoferrin prevents Mozzarella cheese blue discoloration caused by Pseudo- 
monas fluorescens. Food Microbiol. 46:15-24. https://doi.org/10 .1016/j.fm.2014.06.021.

Carroll, R. 2002. Home Cheese Making: Recipes for 75 Homemade Cheeses. Storey Publishing, LLC, North Adams, MA.

Cenci-Goga, B. T., M. Karama, P. Sechi, M. F. Iulietto, S. Novelli, and S. Mattei. 2014. Evolution under different storage conditions of anomalous blue coloration of Mozzarella cheese intentionally contaminated with a pigment-producing strain of Pseudomonas fluorescens. J. Dairy Sci. 97:6708-6718. https://doi.org/10.3168/ jds.2014-8611.

Cha, J. S., and D. A. Cooksey. 1991. Copper resistance in Pseudomonas syringae mediated by periplasmic and outer membrane proteins. Proc. Natl. Acad. Sci. USA 88:8915-8919. https://doi.org/ 10.1073/pnas.88.20.8915

Chierici, M., C. Picozzi, M. G. La Spina, C. Orsi, I. Vigentini, V. Zambrini, and R. Foschino. 2016. Strain diversity of Pseudomonas fluorescens group with potential blue pigment phenotype isolated from dairy products. J. Food Prot. 79:1430-1435. https://doi.org/ 10.4315/0362-028X.JFP-15-589.

Cole, J. R., Q. Wang, E. Cardenas, J. Fish, B. Chai, R. J. Farris, A. S. Kulam-Syed-Mohideen, D. M. McGarrell, T. Marsh, G. M. Garrity, and J. M. Tiedje. 2009. The Ribosomal Database Project: improved alignments and new tools for rRNA analysis. Nucleic Acids Res. 37:D141-D145. https://doi.org/10.1093/nar/gkn879.

Conn, H. W. 1892. The fermentation of milk. USDA Experiment Station Bulletin 9:7-75.

de Vries, A., and B. D. Ripley. 2016. ggdendro: Create dendrograms and tree diagrams using 'ggplot2'. R package version $0.1-20$. https: //CRAN.R-project.org/package $=$ ggdendro.

del Olmo, A., J. Calzada, and M. Nuñez. 2018. The blue discoloration of fresh cheeses: a worldwide defect associated to specific contamination by Pseudomonas fluorescens. Food Control 86:359-366. https://doi.org/10.1016/j.foodcont.2017.12.001.

Duckwall, E. W. 1905. Canning and Preserving of Food Products with Bacteriological Technique. Pittsburgh Printing Company, Pittsburgh, PA.

Dunstall, G., M. T. Rowe, G. B. Wisdom, and D. Kilpatrick. 2005. Effect of quorum sensing agents on the growth kinetics of Pseudomonas spp. of raw milk origin. J. Dairy Res. 72:276-280. https:// doi.org/10.1017/S0022029905000713.

Edgar, R. C. 2004. MUSCLE: multiple sequence alignment with high accuracy and high throughput. Nucleic Acids Res. 32:1792-1797. https://doi.org/10.1093/nar/gkh340.

Ensley, B. D., B. J. Ratzkin, T. D. Osslund, M. J. Simon, L. P. Wackett, and D. T. Gibson. 1983. Expression of naphthalene oxidation genes in Escherichia coli results in the biosynthesis of indigo. Science 222:167-169. https://doi.org/10.1126/science.6353574.

Evanowski, R. L., S. J. Reichler, D. J. Kent, N. H. Martin, K. J. Boor, and M. Wiedmann. 2017. Short communication: Pseudomonas azotoformans causes gray discoloration in HTST fluid milk. J. Dairy Sci. 100:7906-7909. https://doi.org/10.3168/jds.2017-12650.

Finn, R. D., P. Coggill, R. Y. Eberhardt, S. R. Eddy, J. Mistry, A. L. Mitchell, S. C. Potter, M. Punta, M. Qureshi, A. Sangrador-Vegas, G. A. Salazar, J. Tate, and A. Bateman. 2016. The Pfam protein families database: towards a more sustainable future. Nucleic Acids Res. 44(D1):D279-D285. https://doi.org/10.1093/nar/gkv1344.

Food Marketing Institute. 1997. Consumer Attitudes and the Supermarket, 1997. Food Marketing Institute, Arlington, VA.

Food Marketing Institute. 2000. Consumer Attitudes and the Supermarket, 2000. Food Marketing Institute, Arlington, VA.

Food Marketing Institute. 2017. US Grocery Shopper Trends 2017. Food Marketing Institute, Arlington, VA.

Gardner, S. N., T. Slezak, and B. G. Hall. 2015. kSNP3.0: SNP detection and phylogenetic analysis of genomes without genome alignment or reference genome. Bioinformatics 31:2877-2878. https:// doi.org/10.1093/bioinformatics/btv271.

Garrido-Sanz, D., J. P. Meier-Kolthoff, M. Göker, M. Martín, R. Rivilla, and M. Redondo-Nieto. 2016. Genomic and genetic diversity within the Pseudomonas fluorescens complex. PLoS One 11:e0150183. https://doi.org/10.1371/journal.pone.0150183.
Gomila, M., A. Peña, M. Mulet, J. Lalucat, and E. García-Valdés. 2015. Phylogenomics and systematics in Pseudomonas. Front. Microbiol. 6. https://doi.org/10.3389/fmicb.2015.00214.

Greenhalgh, M. . 2010. Bacterial contamination caused blue Mozzarella. Food Safety News. Accessed Dec. 19, 2018, https://www .foodsafetynews.com/2010/06/bacterial-contamination-caused -blue-mozzarella/.

Hammer, B. W. 1914. A bacteriological study of blue milk. Iowa Agricultural Experiment Station Research Bulletin 15:467-481.

Hammer, B. W. 1957. Milk fermentations. Pages 63-115 in Dairy Bacteriology. 4th ed. Wiley, New York, NY.

Haynes, W. C. 1961. Correct description of Pseudomonas mildenbergii Bergey et al., 1930. J. Bacteriol. 81:385-386.

Hueppe, F. 1884. Untersuchungen über die zersetzungen der milch durch mikroorganismen. Mittheilungen aus dem Kaiserlichen Gesundheitsamte 2:309-371.

Hulcher, F. H. 1982. Isolation and characterization of a new hydroxamic acid from Pseudomonas mildenbergii. Biochemistry 21:4491-4495. https://doi.org/10.1021/bi00261a046.

Jones, D. T., W. R. Taylor, and J. M. Thornton. 1992. The rapid generation of mutation data matrices from protein sequences. Comput. Appl. Biosci. 8:275-282. https://doi.org/10.1093/bioinformatics/8 .3.275.

Karp, G. 2010. Capri Sun mold case shows bloggers can take on corporations. Chicago Tribune. Accessed May 6, 2019. https:// www.chicagotribune.com/business/ct-xpm-2010-06-15-ct-biz-0616 -kraft-mold-20100615-story.html.

Kosa, K. M., S. C. Cates, S. Karns, S. L. Godwin, and D. Chambers. 2007. Consumer home refrigeration practices: Results of a web-based survey. J. Food Prot. 70:1640-1649. https://doi.org/10 .4315/0362-028x-70.7.1640.

Kovac, J., R. A. Miller, L. M. Carroll, D. J. Kent, J. Jian, S. M. Beno, and M. Wiedmann. 2016. Production of hemolysin BL by Bacillus cereus group isolates of dairy origin is associated with whole genome phylogenetic clade. BMC Genomics 17:581. https://doi.org/ 10.1186/s12864-016-2883-z.

Kumar, S., G. Stecher, M. Li, C. Knyaz, and K. Tamura. 2018. MEGA $\mathrm{X}$ : molecular evolutionary genetics analysis across computing platforms. Mol. Biol. Evol. 35:1547-1549. https://doi.org/10.1093/ molbev/msy096.

Lagesen, K., P. Hallin, E. A. Rødland, H.-H. Stærfeldt, T. Rognes, and D. W. Ussery. 2007. RNAmmer: consistent and rapid annotation of ribosomal RNA genes. Nucleic Acids Res. 35:3100-3108. https: //doi.org/10.1093/nar/gkm160.

Lindsay, R. C. 1996. Food additives. Pages $767-824$ in Food Chemistry 3rd ed. O. R. Fennema, ed. CRC Press, New York, NY.

Martin, N. H., S. C. Murphy, R. D. Ralyea, M. Wiedmann, and K. J. Boor. 2011. When cheese gets the blues: Pseudomonas fluorescens as the causative agent of cheese spoilage. J. Dairy Sci. 94:31763183. https://doi.org/10.3168/jds.2011-4312.

Mildenberg, H. 1922. Über einen blauen farbstoff bildenden Bacillus aus der luft und seine beziehungen zum Bacillus der blauen milch. Zentralblatt für Bakteriologie. Parasitenkunde und Infektionskrankheiten 56:309-328.

Neilands, J. B. 1967. Hydroxamic acids in nature. Science 156:14431447. https://doi.org/10.1126/science.156.3781.1443.

Page, A. J., C. A. Cummins, M. Hunt, V. K. Wong, S. Reuter, M. T. G. Holden, M. Fookes, D. Falush, J. A. Keane, and J. Parkhill. 2015. Roary: Rapid large-scale prokaryote pan genome analysis. Bioinformatics 31:3691-3693. https://doi.org/10.1093/bioinformatics/ btv 421 .

Palleroni, N. J. 2015. Pseudomonas. In Bergey's Manual of Systematics of Archaea and Bacteria. W. B. Whitman, F. Rainey, P. Kämpfer, M. Trujillo, J. Chun, P. DeVos, B. Hedlund, and S. Dedysh, ed. Wiley, Hoboken, NJ. https://doi.org/10.1002/9781118960608 gbm01210.

Parmentier, A., and N. Deyeux. 1799. Précis d'expériences et observations sur les differentes espèces de lait, eonsidérées dansleurs rapports avec la chimie, la médecine et l'economie rurale. Chez F.G. Levrault, Strasbourg. 
Peix, A., M.-H. Ramírez-Bahena, and E. Velázquez. 2018. The current status on the taxonomy of Pseudomonas revisited: An update. Infect. Genet. Evol. 57(Suppl. C):106-116. https://doi.org/10.1016/ j.meegid.2017.10.026.

Philson, S. B., and M. Llinás. 1982. Siderochromes from Pseudomonas fluorescens. I. Isolation and characterization. J. Biol. Chem. $257: 8081-8085$.

R Core Team. 2018. R: A language and environment for statistical computing. R Foundation for Statistical Computing, Vienna, Austria.

Ranieri, M. L., and K. J. Boor. 2009. Short communication: bacterial ecology of high-temperature, short-time pasteurized milk processed in the United States. J. Dairy Sci. 92:4833-4840. https:// doi.org/10.3168/jds.2009-2181.

Reichler, S. J., A. Trmčić, N. H. Martin, K. J. Boor, and M. Wiedmann. 2018. Pseudomonas fluorescens group bacterial strains are responsible for repeat and sporadic postpasteurization contamination and reduced fluid milk shelf life. J. Dairy Sci. 101:7780-7800. https://doi.org/10.3168/jds.2018-14438.

Richter, M., and R. Rosselló-Móra. 2009. Shifting the genomic gold standard for the prokaryotic species definition. Proc. Natl Acad. Sci. USA 106:19126-19131. https://doi.org/10.1073/pnas .0906412106

Sechi, P., A. Vizzani, S. Scuota, A. Zicavo, S. Parmegiani, and B. C. Goga. 2011. Anomalous blue colouring of Mozzarella cheese intentionally contaminated with pigment producing strains of Pseudomonas fluorescens. Ital. J. Food Saf. 1:81-84. https://doi.org/10 .4081/ijfs.2011.757.

Seemann, T. 2014. Prokka: rapid prokaryotic genome annotation. Bioinformatics 30:2068-2069. https://doi.org/10.1093/bioinformatics/ btu153.

Seiler, H. 2006. Blaue Milch — gibt es die wirklich? Deutsche MolkereiZeitung 12:25-27.

Shirahata, K., T. Deguchi, T. Hayashi, I. Matsubara, and T. Suzuki. 1970. The structures of fluopsins C and F. J. Antibiot. (Tokyo) 23:546-550. https://doi.org/10.7164/antibiotics.23.546.

Stamatakis, A. 2014. RAxML version 8: A tool for phylogenetic analysis and post-analysis of large phylogenies. Bioinformatics 30:13121313. https://doi.org/10.1093/bioinformatics/btu033.
Stones, K. 2013. The era of immediacy-Part II. Accessed December 19, 2018, https://callahan.agency/lessons-from-chobani-greek -yogurt-about-the-era-of-immediacy/.

Trmčić, A., N. H. Martin, K. J. Boor, and M. Wiedmann. 2015. A standard bacterial isolate set for research on contemporary dairy spoilage. J. Dairy Sci. 98:5806-5817. https://doi.org/10.3168/jds $.2015-9490$

USDA Agricultural Research Service. 2018. Northern Regional Research Laboratory (NRRL) Database, National Center for Agricultural Research. Accessed December 19, 2018, https://nrrl.ncaur .usda.gov/cgi-bin/usda/index.html.

Van Tassell, J. A., N. H. Martin, S. C. Murphy, M. Wiedmann, K. J. Boor, and R. A. Ivy. 2012. Evaluation of various selective media for the detection of Pseudomonas species in pasteurized milk. J. Dairy Sci. 95:1568-1574. https://doi.org/10.3168/jds.2011-4958.

Vangay, P., E. B. Fugett, Q. Sun, and M. Wiedmann. 2013. Food microbe tracker: a web-based tool for storage and comparison of food-associated microbes. J. Food Prot. 76:283-294. https://doi .org/10.4315/0362-028X.JFP-12-276.

von Neubeck, M., C. Huptas, C. Glück, M. Krewinkel, M. Stoeckel, T. Stressler, L. Fischer, J. Hinrichs, S. Scherer, and M. Wenning. 2017. Pseudomonas lactis sp. nov. and Pseudomonas paralactis sp. nov., isolated from bovine raw milk. Int. J. Syst. Evol. Microbiol. 67:1656-1664. https://doi.org/10.1099/ijsem.0.001836.

Whitaker, J. R. 1996. Enzymes. Pages 431-530 in Food Chem. 3rd ed. O. R. Fennema, ed. CRC Press, New York, NY.

Wickham, H. 2016. ggplot2: Elegant graphics for data analysis. 2nd ed. Springer International Publishing, New York

Yam, K. L., and S. E. Papadakis. 2004. A simple digital imaging method for measuring and analyzing color of food surfaces. J. Food Eng. 61:137-142. https://doi.org/10.1016/S0260-8774(03)00195-X.

Zakataeva, N. P., V. V. Aleshin, I. L. Tokmakova, P. V. Troshin, and V. A. Livshits. 1999. The novel transmembrane Escherichia coli proteins involved in the amino acid efflux. FEBS Lett. 452:228 232. https://doi.org/10.1016/S0014-5793(99)00625-0. 